\title{
Quantum Principal Bundles on Projective Bases
}

\author{
Paolo Aschieri ${ }^{1,2,3}$ (D) , Rita Fioresi ${ }^{4}$, Emanuele Latini ${ }^{4,5}$ \\ ${ }^{1}$ Dipartimento di Scienze e Innovazione Tecnologica, Università del Piemonte Orientale, Viale T. Michel, \\ 15121 Alessandria, Italy. E-mail: paolo.aschieri@uniupo.it \\ 2 Istituto Nazionale di Fisica Nucleare, Sezione di Torino, Via P. Giuria 1, 10125 Turin, Italy \\ 3 Arnold-Regge Center, Torino, Via P. Giuria 1, 10125 Turin, Italy \\ 4 Dipartimento di Matematica, Università di Bologna, Piazza di Porta S. Donato 5, 40126 Bologna, Italy. \\ E-mail: rita.fioresi@UniBo.it \\ 5 Istituto Nazionale di Fisica Nucleare, Sezione di Bologna, Viale Berti Pichat 6/2, 40127 Bologna, Italy. \\ E-mail: emanuele.latini@UniBo.it
}

\begin{abstract}
The purpose of this paper is to propose a sheaf theoretic approach to the theory of quantum principal bundles over non affine bases. We study noncommutative principal bundles corresponding to $G \rightarrow G / P$, where $G$ is a semisimple group and $P$ a parabolic subgroup.
\end{abstract}

\section{Contents}

1. Introduction

2. Quantum Principal Bundles

2.1 The classical description

2.2 The quantum description

3. Quantum Homogenous Projective Varieties

3.1 Projective embeddings of homogeneous spaces

3.2 Quantum homogeneous projective varieties and quantum sections

4. Quantum Principal Bundles from Parabolic Quotients $G / P \ldots \ldots$. . .

4.1 Sheaves of comodule algebras

4.2 Quantum principal bundles on quantum homogeneous spaces . . . . .

5. Examples on Quantum Projective Spaces . . . . . . . . . . . . . .

5.1 Quantum deformations of function algebras

5.2 Quantum principal bundles on quantum projective spaces . . . . . . .

6. Quantum Principal Bundles from Twists . . . . . . . . . . . .

6.1 Deformations from twists of $H \ldots \ldots \ldots \ldots$

6.2 Deformations from twists of $K$

6.3 Examples 


\section{Introduction}

A quantum principal bundle is usually described as an algebra extension $B \subset A$, with $A$ the "total space" algebra on which acts a quantum group, and $B$ the "base space" subalgebra of invariant elements. Local triviality is encompassed as a special case in the notion of locally cleft extension.

In the commutative setting, this picture proves to be extremely effective when the base space $M$ is affine, that is, when the algebra $B$ is containing all of the information to reconstruct the base space. For a projective base, however, the algebra $B$ of invariants under the group action consists of just the constants, so it is not the object of interest anymore. The way out in classical algebraic geometry is to localize and consider a sheaf theoretic formulation of the base space and then of bundle theory (where the structure group is replaced by the structure sheaf of germs of functions from the base space to the group), see [22].

In [38] Pflaum gave a sheaf theoretic characterization of noncommutative bundles. This has been further developed in [23] in the context of principal comodule algebras (e.g. Hopf-Galois extensions with cosemisimple structure Hopf algebra) and by considering flabby sheaves i.e. sheaves with surjective restriction maps; this last condition is a sufficient condition in order to glue together (via multipullbacks) principal comodule algebras locally defined on the base space to a globally defined one. In the noncommutative affine case, since the total space algebra is a Hopf-Galois extension and since the Hopf-Galois property restricts locally it is natural to study sheaves of Hopf-Galois extensions [8], see also [2].

In this paper we take a very general point of view on the definition of quantum principal bundle (see Definition 2.9), so that we can accomodate the affine setting mentioned above, but also the case of projective base, together with a preferred projective embedding. In our definition a quantum principal bundle is a locally cleft, and more generally a locally principal, sheaf of $H$ comodule algebras for a given Hopf algebra $H$. The sheaf in general is not flabby. In the commutative setting, when the base is affine the algebra of global sections (regular functions on the total space) is a Hopf-Galois extension; when the base is a projective variety our notion still makes sense and it actually gives the correct point of view to proceed to the quantization.

The definition is tested on an important special case, that when $M$ is the quotient of a semisimple group $G$ by a parabolic subgroup $P$. In this case, in fact, $M=G / P$ is projective, and we can effectively substitute the $P$-invariant ring $B$, i.e., the $\mathcal{O}(P)$ coaction invariant (for short coinvariant) ring $B$, with the homogeneous coordinate ring $\tilde{\mathcal{O}}(G / P)$ of $G / P$ with respect to a chosen projective embedding, corresponding to a line bundle $\mathcal{L}$. The line bundle $\mathcal{L}$ can be recovered more algebraically via a character $\chi$ of $P$; the corresponding sections are the $\mathcal{O}(P)$-semi-coinvariant elements of $\mathcal{O}(G)$ with respect to $\chi$ and generate the homogeneous coordinate $\operatorname{ring} \tilde{\mathcal{O}}(G / P)$ of $G / P$. In this case the locally cleft sheaf of $H=\mathcal{O}(P)$-comodule algebras, denoted $\mathcal{F}$, gives the subsheaf of coinvariants $\mathcal{F}^{\mathrm{co}} \mathcal{O}(P)$ that is the structure sheaf $\mathcal{O}_{G / P}$ of $G / P$. The relation between this latter and the homogeneous coordinate ring $\tilde{\mathcal{O}}(G / P)$ is then as usual by considering projective localizations (zero degree subalgebras of the localizations) of $\tilde{\mathcal{O}}(G / P)$.

Similarly, in the quantum case, as in $[10,17]$ we obtain the quantum homogeneous coordinate ring $\tilde{\mathcal{O}}_{q}(G / P)$ as the $\mathcal{O}_{q}(P)$-semi-coinvariant elements of the quantum group $\mathcal{O}_{q}(G)$, the quantization of the semisimple group $G$. Assuming Ore conditions for localizations, we then proceed to obtain from $\tilde{\mathcal{O}}_{q}(G / P)$ and $\mathcal{O}_{q}(G)$ a suitable sheaf $\mathcal{F}$ 
of $\mathcal{O}_{q}(P)$-comodule algebras, which will be the quantum principal bundle over the quantum space obtained through $\tilde{\mathcal{O}}_{q}(G / P)$. More explicitly, the coinvariant subsheaf $\mathcal{F}^{\operatorname{co} \mathcal{O}_{q}(P)}$ will be the quantum structure sheaf associated with the (noncommutative) projective localizations of $\tilde{\mathcal{O}}_{q}(G / P)$.

The quantization of the flag variety $G / P$ and its noncommutative geometry has recently attracted a lot of attention. The theory, also following the remarkable classification of differential calculi over irreducible quantum flag manifolds in [26,27], has been conspicuously developed in the past years, see for example [11,12,28, 29,33,34]. In particular, the study of quantum projective space as a quantum homogeneous space has proven fruitful, however, it has mainly concerned quantum projective space as the base space of a quantum principal $U(N-1)$-bundle with quantum $S U(N)$ total space, i.e., a study not in the projective context. Indeed, despite the progress on quantum principal bundles $[4,6,7,23]$, the projective setting, describing quantum versions of principal bundles $G \rightarrow G / P$, with $P$ parabolic, is yet to be fully understood. The aim of this paper is to provide a key step in this direction, together with an appropriate setting for a future differential calculus and theory of principal connections on such quantizations.

The approach we develop turns out to be a rather conservative one with respect to the fundamental results in noncommutative algebraic geometry $[1,19,36]$; for example we localize using Ore condition [41]. In [1], inspired by Serre's equivalence between the categories of quasi-coherent sheaves on a projective scheme $X=\operatorname{Proj} A$ and that of a quotient category of the category of graded $A$-modules, the authors develop a general theory by defining a quantum projective scheme as a category of modules associated with a graded noncommutative algebra. In our approach, we start by the quantization of the homogeneous coordinate ring of an homogeneous space, which is exactly the graded noncommutative algebra $A$ in [1], however in a more specific case, namely when $X$ is an homogeneous algebraic space.

Also in [19,36] the authors take a categorical point of view. In [36], an affine quantum space is viewed as the spectrum of a noncommutative ring, where the notion of spectrum is defined appropriately (see $[36, \S 1]$ ); projective quantum schemes are then defined accordingly (see [36, §VII.2]) through a generalization of the classical Proj construction and the categorical language is employed to prove a gluing construction of the quantum Proj in terms of quantum affine schemes. Notice that in [36, §VII.5.3] one finds the example of the flag and its graded algebra $\mathcal{R}$, which of course coincides with the description in [10], which is the starting point for our present treatment.

In [19] the authors define noncommutative quasi-projective schemes using the category of affine covers, whose morphisms give the notion of refinement (see [19, $\S 2])$. In this way they ensure that compatibility conditions, like the Ore for subsequent localization (see $[41, \S \mathrm{IV}, \S \mathrm{V}]$ ), are automatically satisfied.

We summarize the main results by explaining the organization of the paper.

In Sect. 2 we recall basic notions in Hopf-Galois extensions, including the inspiring sheaf approach of $[8,38]$. We then present our sheaf theoretic definition of quantum principal bundle. We also provide the example of $\mathrm{SL}_{2}(\mathbb{C}) / P$ both in the classical and in the quantum setting. This serves also as motivation and preparation for the general theory we develop in later sections.

In Sect. 3 we discuss quantum homogenous projective varieties, mainly following $[10, \S 2]$. Starting from a quantum section $d \in \mathcal{O}_{q}(G)$, quantum version of the lift to $\mathcal{O}(G)$ of the character $\chi$ of $P$ defining the line bundle $\mathcal{L}$ giving the projective embedding of $G / P$, we construct the homogeneous ring $\tilde{\mathcal{O}}_{q}(G / P)$. 
In Sect. 4, we develop a general theory for quantum principal bundles on homogeneous projective varieties. We construct the sheaf $\mathcal{F}$ of $\mathcal{O}_{q}(P)$-comodule algebras on the quantum projective variety $\tilde{\mathcal{O}}_{q}(G / P)$ by local data, that is by considering suitable projective localizations of $\tilde{\mathcal{O}}_{q}(G / P)$, obtained via a corresponding quantum section $d \in \mathcal{O}_{q}(G)$. As shown in Theorem 4.8, if this sheaf is locally cleft we have a quantum principal bundle.

In Sect. 5, we exemplify the construction of Sect. 4 in the case of quantum projective space. We prove that quantum projective space is the base space of a canonical quantum principal bundle with total space $\mathcal{O}_{q}\left(\mathrm{SL}_{n}\right)$ and structure group $\mathcal{O}_{q}(P)$ (quantum parabolic subgroup of $\left.\mathcal{O}_{q}\left(\mathrm{SL}_{n}\right)\right)$.

In Sect. 6, we apply and further develop the results in [2] and show that 2-cocycle deformations (twists) of quantum principal bundles give new quantum principal bundles. We construct three classes of quantum principal bundles on quantum projective spaces. The first two are locally cleft but not locally trivial. The total spaces are not Hopf algebras hence they are not quantum principal bundles on quantum homogenous projective space as in the construction presented in Theorem 4.8. The second and third class are on multiparametric quantum projective space, the third class being also an example of the construction in Theorem 4.8, with total space the multiparametric special linear quantum group.

\section{Quantum Principal Bundles}

In the category of locally compact Hausdorff topological spaces, a principal bundle is a bundle $E \rightarrow M$, with compatibility requirements regarding the $P$-space structure, for a given topological group $P$. These requirements can be effectively summarized by asking that the map

$$
E \times P \longrightarrow E \times{ }_{M} E \quad(e, p) \mapsto(e, e p)
$$

is a homeomorphism, with $M=E / P$ and $E \times{ }_{M} E$ closed in $E \times E$.

We can dualize this picture by replacing spaces with their function algebras. If $E$ and $M$ are affine algebraic varieties and $P$ an affine algebraic group we consider the coordinate rings $A=\mathcal{O}(E), B=\mathcal{O}(M)$ and $H=\mathcal{O}(P)$. The notion of principal bundle is then replaced by that of principal comodule algebra, or equivalently, faithfully flat Hopf-Galois extension. The Hopf-Galois property is the freeness of the $P$-action, and amounts to the requirement that the pullback of the above map, called canonical map,

$$
\chi: A \otimes_{B} A \rightarrow A \otimes H
$$

is a bijection. The faithfully flat property corresponds to the principality of the action (see e.g. [7]).

The contravariant functor associating to affine varieties their coordinate ring is an equivalence of categories (see [25, Proposition 2.6, §II] for more details). When we turn to examine the case of projective varieties, since this equivalence of categories does not hold anymore as stated, but becomes more involved, we need to take a different approach to the theory of principal bundles, introducing the sheaves of functions on our geometric objects. As it turns out, this approach, pioneered in [22], despite its apparent complication and abstraction is very suitable for quantization. Indeed, more generally, the sheaf approach is a key method in noncommutative algebraic geometry [41]. 
2.1. The classical description. We start with a description of the classical setting.

Definition 2.1. Let $E$ and $M$ be topological spaces, $P$ a topological group and $\wp$ : $E \longrightarrow M$ a continuous function. We say that $(E, M, \wp, P)$ is a $P$-principal bundle (or principal bundle for short) with total space $E$ and base $M$, if the following conditions hold

1. $\wp$ is surjective.

2. $P$ acts freely from the right on $E$.

3. $P$ acts transitively on the fiber $\wp^{-1}(m)$ of each point $m \in M$.

4. $E$ is locally trivial over $M$, i.e. there is an open covering $M=\cup U_{i}$ and homeomorphisms $\sigma_{i}: \wp^{-1}\left(U_{i}\right) \longrightarrow U_{i} \times P$ that are $P$-equivariant i.e., $\sigma_{i}(u p)=\sigma(m) p$, $p \in P$.

We can speak of algebraic, analytic or smooth $P$-principal bundles, we just take the objects and the morphism of Definition 2.1 in the appropriate categories. Notice that $\wp$ is open.

In [38] Pflaum gives a sheaf theoretic characterization of principal bundles, in the category of locally compact topological spaces, which is very suitable for noncommutative geometry.

In the algebraic category, over a field $k$, we can give another characterization of principal bundles, closely related to Pflaum's one. For the basic definitions regarding algebraic groups we refer e.g. to [3, §II], for Hopf algebras e.g. to [31], [5, Part VII §5 ].

Proposition 2.2. Let $\wp: E \longrightarrow M$ be a surjective morphism of algebraic varieties, and $\mathcal{O}_{E}, \mathcal{O}_{M}$ the structural sheaves of $E$ and $M$ respectively. Let $\mathcal{F}$ be the sheaf on $M$ defined by $\mathcal{F}(U)=\mathcal{O}_{E}\left(\wp^{-1}(U)\right)$. Let $P$ be an affine algebraic group, $H$ the associated Hopf algebra. Then $E \longrightarrow M$ is a principal bundle if and only if

- $\mathcal{F}$ is a sheaf of $H$ comodule algebras: for each open $U \subset M, \mathcal{F}(U)$ is a right $H$-comodule algebra and for each open $W \subset U$ the restriction map $r_{U W}: \mathcal{F}(U) \rightarrow$ $\mathcal{F}(W)$ is a morphism of $H$-comodule algebras;

- There exists an open covering $\left\{U_{i}\right\}$ of $M$ such that we have the following algebra isomorphisms for all $i$

1. $\mathcal{F}\left(U_{i}\right)^{\operatorname{co} H} \simeq \mathcal{O}_{M}\left(U_{i}\right)$,

2. $\mathcal{F}\left(U_{i}\right) \simeq \mathcal{F}\left(U_{i}\right)^{\mathrm{co} H} \otimes H$, where $\mathcal{F}\left(U_{i}\right)^{\mathrm{co} H}:=\left\{f \in \mathcal{F}\left(U_{i}\right) \mid \delta(f)=f \otimes 1\right\} \subset \mathcal{F}\left(U_{i}\right)$ is the subalgebra of $H$-coinvariant elements, with $\delta: \mathcal{F}\left(U_{i}\right) \rightarrow \mathcal{F}\left(U_{i}\right) \otimes H$ the $H$-coaction.

We notice that condition (1) establishes $M \simeq E / P$; we will identify $M$ and $E / P$, so that correspondingly $\mathcal{F}\left(U_{i}\right)^{\mathrm{co} H}=\mathcal{O}_{M}\left(U_{i}\right)$. Condition (2) gives the local triviality, the transitive action of $P$ on the fiber and the freeness of the $P$ action on $E$. We leave the details of this characterization to the reader, it will be a small variation of the argument given in [38].

2.2. The quantum description. We now proceed and extend this point of view in order to give the definition of quantum principal bundle: it is based on [38] (see also Proposition 2.2) and also on [8], but it is more general since it encompasses the possibility for the base manifold to be projective. Furthermore, we take our category to be algebraic.

We will work with algebras (not necessarily commutative) over a field $k$ of characteristic 0 , or the ring of Laurent polynomials $k_{q}=k\left[q, q^{-1}\right], q$ an indeterminate. All 
algebras will be unital and morphisms preserve the unit. In particular we will work with $H$-comodule algebras $(A, \delta)$, where $\delta$ denotes the Hopf algebra coaction (frequently omitted). Hopf algebras will be with bijective antipode.

Definition 2.3. Let $(H, \Delta, \varepsilon, S)$ be a Hopf algebra and $A$ be an $H$-comodule algebra with coaction $\delta: A \longrightarrow A \otimes H$. Let

$$
B:=A^{\mathrm{co} H}:=\{a \in A \mid \delta(a)=a \otimes 1\} .
$$

The extension $A$ of the algebra $B$ is called H-Hopf-Galois (or simply Hopf-Galois) if the map

$$
\chi: A \otimes_{B} A \longrightarrow A \otimes H, \quad \chi=\left(m_{A} \otimes i d\right)\left(i d \otimes_{B} \delta\right)
$$

(called the canonical map) is bijective.

The extension $B=A^{\mathrm{co} H} \subset A$ is called $H$-principal comodule algebra if it is HopfGalois and $A$ is $H$-equivariantly projective as a left $B$-module, i.e., there exists a left $B$-module and right $H$-comodule morphism $s: A \rightarrow B \otimes A$ that is a section of the (restricted) product $m: B \otimes A \rightarrow A$.

If $E \longrightarrow M$ is a $P$-principal bundle and $E, M$ and $P$ are affine algebraic varieties then the algebra of coordinate functions on $E$ and $P$ correspond respectively to the algebras $A$ and $H$ satisfying Definition 2.3. The algebra $B$ is the algebra of functions on the base manifold $M$.

We denote as usual by $\ell * j$ the convolution product of two linear maps $j: H \rightarrow A$, $\ell: H \rightarrow A$. It is defined by $\ell * j(h)=\ell\left(h_{1}\right) j\left(h_{2}\right)$ for all $h \in H$. A linear map $j: H \rightarrow A$ is convolution invertible if it exists $j^{-1}: H \rightarrow A$ such that $j^{-1} * j=$ $j * j^{-1}: H \rightarrow A, h \mapsto \varepsilon(h) 1_{A}$. If $A$ is a right $H$-comodule we can require $j: H \rightarrow A$ to be a right $H$-comodule map where $H$ has $H$-comodule structure given by $\Delta$, i.e., $\delta \circ j=(j \otimes i d) \circ \Delta$.

Definition 2.4. Let $H$ be a Hopf algebra and $A$ an $H$-comodule algebra. The algebra extension $A^{\text {co } H} \subset A$ is called a cleft extension if there is a right $H$-comodule map $j: H \rightarrow A$, called cleaving map, that is convolution invertible.

An extension $A^{\text {co } H} \subset A$ is called a trivial extension if there is an $H$-comodule algebra map $j: H \rightarrow A$.

Since $1_{H}$ is a grouplike element $j\left(1_{H}\right) j^{-1}\left(1_{H}\right)=1_{A}$, so that $j\left(1_{H}\right)$ is an invertible element in $A^{\text {co } H}$. Hence a cleaving map can always be normalised to $j\left(1_{H}\right)=1_{A}$. We will always consider normalized cleaving maps.

Remark 2.5. A trivial extension $A^{\text {co } H} \subset A$ is automatically a cleft extension. In fact, since an $H$-comodule algebra map $j: H \rightarrow A$ maps the unit of $H$ in that of $A$, its convolution inverse is $j^{-1}=j \circ S$. Furthermore, the $H$-comodule algebra map $j: H \rightarrow A$ is an injection, indeed the map $(\varepsilon \otimes i d) \circ(m \otimes i d) \circ(i d \otimes j \circ S \otimes i d) \circ(\delta \otimes i d) \circ \delta$ sends $j(h)$ to $h$. Thus the subalgebra $j(H) \subset A$ is isomorphic to $H$.

By a theorem of Doi and Takeuchi [13] (we also refer to [31, Theorem 8.2.4], [5, Part VII §5]) cleft extensions are special cases of Hopf-Galois extensions. They are furthermore special cases of principal comodule algebras (see e.g. [5, Part VII §6], [7]).

Theorem 2.6. Let $A$ be an $H$-comodule algebra with base ring a field $k$, then $A^{\text {co } H} \subset A$ is a cleft extension if and only if $A^{\mathrm{co} H} \subset A$ is a Hopf-Galois extension and there is an $H$-comodule and left $B=A^{\text {co } H}$-module isomorphism $B \otimes H \simeq A$. Furthermore, this Hopf-Galois extension is a principal comodule algebra. 
Here $B \otimes H$ is an $H$-comodule with $H$-coaction $i d \otimes \Delta$, the extension $B \subset B \otimes H$ is easily seen to be Hopf-Galois and is an example of trivial extension (with $j(h)=1_{B} \otimes h$, for all $h \in H)$. For later use we recall that the relation betweeen a cleaving map $j: H \rightarrow$ $A$ and the left $B=A^{\text {co } H}$-module and $H$-comodule isomorphism $\theta: B \otimes H \rightarrow A$ is given by $\theta(b \otimes h)=b j(h)$.

The notion of cleft extension encompasses that of trivial principal bundle. The next observation sharpens the relation between trivial Hopf-Galois extensions, trivial principal bundles and cleft extensions.

Observation 2.7. If $j: H \rightarrow A$ is an $H$-comodule algebra map, then we have an action of $H$ on $B=A^{\text {co } H}$ given by $h \triangleright b=j\left(h_{1}\right) b j^{-1}\left(h_{2}\right)=j\left(h_{1}\right) b j\left(S\left(h_{2}\right)\right)$, for all $h \in H, b \in B$. We can therefore consider the smashed product algebra $B \sharp H$, that is the $H$-comodule $B \otimes H$ with product structure $(b \otimes h)\left(b^{\prime} \otimes h^{\prime}\right)=b\left(h_{1} \triangleright b^{\prime}\right) \otimes h_{2} h^{\prime}$. With this product $\theta: B \sharp H \rightarrow A$ is an $H$-comodule algebra isomorphism. If $B$ is central the smashed product is the usual tensor product of algebras. In particular, in the affine case, we immediately recover that a $P$-principal bundle $E \rightarrow E / P$ is trivial if and only if $\mathcal{O}(E / P) \otimes \mathcal{O}(P) \simeq \mathcal{O}(E)$ as $\mathcal{O}(P)$-comodule algebras.

In the more general case of an extension that is nontrivial but cleft, the map $j: H \rightarrow A$ is not an $H$-comodule algebra map, and the 2-cocycle

$$
\tau: H \otimes H \rightarrow B, \quad \tau(h, k)=j\left(h_{(1)}\right) j\left(k_{(1)}\right) j^{-1}\left(h_{(2)} k_{(2)}\right)
$$

measures this failure. In general the map $h \otimes b \mapsto j\left(h_{1}\right) b j^{-1}\left(h_{2}\right)$ is not an action of $H$ on $B$. In this cleft case we can still induce via the isomorphism $\theta: B \otimes H \rightarrow A$ an algebra structure on $B \otimes H$, this corresponds to a crossed product $B \sharp_{\tau} H$ (see e.g. [31, Proposition 7.2.3]).

We want to present a notion of quantum principal bundle that is more general than that of Hopf-Galois extension presented in Definition 2.3, and which can accomodate also the case where $M$ is an algebraic variety, which is not affine. To this end, we consider a sheaf theoretic description of quantum principal bundles. We start by introducing the notion of quantum ringed space.

Definition 2.8. A quantum ringed space $\left(M, \mathcal{O}_{M}\right)$ is a pair consisting of a classical topological space $M$ and a sheaf over $M$ of noncommutative algebras.

Classical differentiable manifolds or algebraic varieties, together with the sheaves of functions on them (differentiable or algebraic) are examples of quantum ringed spaces. Also supergeometry provides important examples (see [9] Ch. 3). We now define the key notions of locally cleft quantum principal bundle and of quantum principal bundle by first extending to the quantum case what we established in Proposition 2.2 and then generalizing it by considering sheaves of $H$-comodule algebras that are locally principal rather than locally cleft.

Definition 2.9. Let $\left(M, \mathcal{O}_{M}\right)$ be a ringed space and $H$ a Hopf algebra. We say that a sheaf of $H$-comodule algebras $\mathcal{F}$ is an $H$-locally cleft principal bundle or locally cleft quantum principal bundle over $\left(M, \mathcal{O}_{M}\right)$ if there exists an open covering $\left\{U_{i}\right\}$ of $M$ such that:

1. $\mathcal{F}\left(U_{i}\right)^{\mathrm{co} H}=\mathcal{O}_{M}\left(U_{i}\right)$,

2. $\mathcal{F}$ is locally cleft, that is, $\mathcal{F}\left(U_{i}\right)$ is a cleft extension of $\mathcal{F}\left(U_{i}\right)^{\operatorname{co} H}$.

The locally cleft property is equivalent to the existence of a projective cleaving map that is a collection of cleaving maps $j_{i}: H \longrightarrow \mathcal{F}\left(U_{i}\right)$. 
The above definition is in the spirit of a Steenrod approach to principal bundles. If we follow the Cartan approach, we are led to consider principal $H$-comodule algebras in the noncommutative affine case, and we are then led to the following generalization.

Definition 2.10. We define a sheaf $\mathcal{F}$ of $H$-comodule algebras to be an $H$-principal bundle or quantum principal bundle over $\left(M, \mathcal{O}_{M}\right)$ by replacing condition 2 in Definition 2.9 with

$2^{\prime} . \mathcal{F}$ is locally principal, that is, $\mathcal{F}\left(U_{i}\right)$ is a principal $H$-comodule algebra.

Recalling the second part of Theorem 2.6, we see that if the base ring $k$ is a field, condition 2 implies condition $2^{\prime}$, hence locally cleft quantum principal bundles are quantum principal bundles.

In the special case that the sheaf $\mathcal{F}$ is locally principal and flabby, it follows from [23, Corollary 3.10] that $\mathcal{F}(M)$ is a principal $H$-comodule algebra.

If, on the other hand, as in [8], we consider $\mathcal{F}$ to be a sheaf of $H$-Hopf-Galois extensions we see, since such a sheaf is locally cleft if it so as a sheaf of $H$-comodule algebras, that a locally cleft sheaf $\mathcal{F}$ of Hopf-Galois extensions is in particular a quantum principal bundle on the quantum ringed space $\left(M, \mathcal{F}^{\mathrm{co} H}\right)$. Even more, a quantum principal bundle $\mathcal{F}$ on the quantum ringed space $\left(M, \mathcal{F}^{\mathrm{co} H}\right)$ has the property $\mathcal{O}_{M}(M)=\mathcal{F}(M)^{\mathrm{co} H} \subset \mathcal{F}(M)$ is Hopf-Galois, if and only if it is a locally cleft sheaf of Hopf-Galois extensions; indeed, as observed in [8], the property of being Hopf-Galois restricts locally.

Let us see a simple example, in the commutative setting, that we will generalize to the noncommutative setting and generic dimensions.

Example 2.11. Let $E=\mathrm{SL}_{2}(\mathbb{C})$ and consider the principal bundle $\wp: \mathrm{SL}_{2}(\mathbb{C}) \longrightarrow$ $\mathrm{SL}_{2}(\mathbb{C}) / P \simeq \mathbf{P}^{1}(\mathbb{C})$, where $P$ is the upper Borel in $\mathrm{SL}_{2}(\mathbb{C})$, i.e., the subgroup of all matrices with vanishing entry $(1,2)$. Let $A=\mathcal{O}\left(\mathrm{SL}_{2}\right)$ be the algebra of regular functions on the complex special linear group $\mathrm{SL}_{2}(\mathbb{C})$. We explicitly have

$$
\mathcal{O}\left(\mathrm{SL}_{2}\right)=\mathbb{C}[a, b, c, d] /(a d-b c-1),
$$

where $\mathbb{C}[a, b, c, d]$ denotes the commutative algebra over $\mathbb{C}$ freely generated by the symbols $a, b, c, d$, while $(a d-b c-1)$ denotes the ideal generated by the element $a d-b c-1$, that implements the determinant relation.

Let $\mathcal{O}(P)$ be the algebra of functions on $P \subset \mathrm{SL}_{2}(\mathbb{C})$, this is the quotient $\mathcal{O}\left(\mathrm{SL}_{2}\right) /(c)=$ $\mathbb{C}\left[t, p, t^{-1}\right]:=\mathbb{C}[t, p, s] /(t s-1)$. With the comultiplication $\Delta$ in $\mathcal{O}\left(\mathrm{SL}_{2}\right)$ and the projection

$$
\pi: \mathcal{O}\left(\mathrm{SL}_{2}\right) \longrightarrow \mathcal{O}\left(\mathrm{SL}_{2}\right) /(c)
$$

that on the generators reads $\left(\begin{array}{ll}a & b \\ c & d\end{array}\right) \mapsto\left(\begin{array}{ll}t & p \\ 0 & t^{-1}\end{array}\right)$ (and is extended as an algebra map) we can define the coaction

$$
\delta=(i d \otimes \pi) \Delta: \mathcal{O}\left(\mathrm{SL}_{2}\right) \rightarrow \mathcal{O}\left(\mathrm{SL}_{2}\right) \otimes \mathcal{O}(P) .
$$

The coinvariants $B=A^{\operatorname{co} \mathcal{O}(P)}$ of this coaction are just the constants, indeed the coinvariant are functions on the base space $\mathbf{P}^{1}(\mathbb{C})$, and the only regular functions on all projective space are the constants (Liouville theorem). We see that the extension $A^{\text {co } \mathcal{O}(P)} \subset A$ is not Hopf-Galois, and that this is due to the lack of regular functions on the base space of the $P$-principal bundle $\wp: \mathrm{SL}_{2}(\mathbb{C}) \rightarrow \mathrm{SL}_{2}(\mathbb{C}) / P \simeq \mathbf{P}^{1}(\mathbb{C})$. 
Nevertheless, we can define an $\mathcal{O}(P)$-principal bundle structure according to Definition 2.9. To this aim, we first consider an affine open cover of the total space and then we project it to the base.

Let $\left\{V_{1}, V_{2}\right\}$ be the open cover of $\mathrm{SL}_{2}(\mathbb{C})$ where $V_{i}$ consists of those matrices in $\mathrm{SL}_{2}(\mathbb{C})$ with entry $(i, 1)$ not equal to zero. Define $U_{i}=\wp\left(V_{i}\right)$ and observe that $\left\{U_{1}, U_{2}\right\}$ is an open cover of $\mathbf{P}^{1}(\mathbb{C})$ since $\wp$ is an open map. The algebras of functions on the open sets $V_{1}$ and $V_{2}$ are the localizations

$$
A_{1}:=\mathcal{O}\left(\mathrm{SL}_{2}\right)\left[a^{-1}\right]=A\left[a^{-1}\right], \quad A_{2}:=\mathcal{O}\left(\mathrm{SL}_{2}\right)\left[c^{-1}\right]=A\left[c^{-1}\right] .
$$

The coaction in (3) uniquely extends to coactions $\delta_{i}: A_{i} \longrightarrow A_{i} \otimes \mathcal{O}(P)$ on these localizations (namely $\delta a^{-1}=a^{-1} \otimes t^{-1}, \delta c^{-1}=c^{-1} \otimes t^{-1}$ ). The coinvariant subalgebras $B_{i}=A_{i}^{\operatorname{co} \mathcal{O}(P)}$ explicitly read

$$
B_{1}=\mathbb{C}\left[a^{-1} c\right] \simeq \mathbb{C}[z], \quad B_{2}=\mathbb{C}\left[a c^{-1}\right] \simeq \mathbb{C}[w] .
$$

Notice that they are the coordinate rings of the affine algebraic varieties $U_{i} \simeq \mathbb{C}$ open in $\mathrm{SL}_{2}(\mathbb{C}) / P \simeq \mathbf{P}^{1}(\mathbb{C})$.

Next we consider on $\mathbf{P}^{1}(\mathbb{C})$ the topology $\left\{\emptyset, U_{12}=U_{1} \cap U_{2}, U_{1}, U_{2}, \mathbf{P}^{1}(\mathbb{C})\right\}$ (this is a rough topology, but sufficient to describe the principal bundle on $\mathbf{P}^{1}(\mathbb{C})$ ). We then define the ringed space $\left(\mathbf{P}^{1}(\mathbb{C}), \mathcal{O}_{\mathbf{P}^{1}(\mathbb{C})}\right)$ with sheaf of regular functions $\mathcal{O}_{\mathbf{P}^{1}(\mathbb{C})}$ given by

$$
\mathcal{O}_{\mathbf{P}^{1}(\mathbb{C})}\left(U_{i}\right):=B_{i}, \quad \mathcal{O}_{\mathbf{P}^{1}(\mathbb{C})}\left(U_{12}\right):=B_{12}:=B_{1}\left[z^{-1}\right], \quad \mathcal{O}_{\mathbf{P}^{1}(\mathbb{C})}\left(\mathbf{P}^{1}(\mathbb{C})\right):=\mathbb{C}
$$

and with $\mathcal{O}_{\mathbf{P}^{1}(\mathbb{C})}(\emptyset)$ being the one element algebra over $\mathbb{C}$, terminal object in the category of algebras. It is easy to verify that the restriction morphism $r_{12,2}: B_{2} \rightarrow B_{12}, w \mapsto z^{-1}$, with all other ones being given by the obvious inclusions (but for the empty set where we have the canonical projections), indeed define the sheaf of regular functions on $\mathbf{P}^{1}(\mathbb{C})$.

Finally we define the sheaf $\mathcal{F}$ of $\mathcal{O}(P)$-comodule algebras

$$
\mathcal{F}\left(U_{i}\right):=A_{i}, \quad \mathcal{F}\left(U_{12}\right):=A_{12}:=A_{1}\left[c^{-1}\right]=A_{2}\left[a^{-1}\right], \quad \mathcal{F}\left(\mathbf{P}^{1}(\mathbb{C})\right)=\mathcal{O}\left(\mathrm{SL}_{2}\right),
$$

and $\mathcal{F}(\emptyset):=\{0\}$ (the one element algebra) with the obvious restriction morphisms.

We now show that all properties required by Definition 2.9 are satisfied. Indeed by construction $\mathcal{O}\left(U_{i}\right)=B_{i}=A_{i}^{\operatorname{co} \mathcal{O}(P)}=\mathcal{F}\left(U_{i}\right)^{\operatorname{co} \mathcal{O}(P)}$. Furthermore the $\mathcal{O}(P)$ comodule $\mathcal{F}\left(U_{1}\right)$ is a trivial extension (and hence a cleft extension) because the map $j_{1}: \mathcal{O}(P) \rightarrow A_{1}$ defined on the generators by

$$
t^{ \pm 1} \mapsto a^{ \pm 1}, \quad p \mapsto b,
$$

and extended as algebra morphism to all $\mathcal{O}(P)$ is well defined and easily seen to be an $\mathcal{O}(P)$-comodule morphism (recall $\delta a^{ \pm 1}=a^{ \pm 1} \otimes t^{ \pm 1}$ and $\delta b=b \otimes t^{-1}+a \otimes p$ ). Similarly, $\mathcal{F}\left(U_{2}\right)$ is a trivial extension with $j_{2}: \mathcal{O}(P) \rightarrow A_{2}$ given by $t^{ \pm 1} \rightarrow c^{ \pm 1}$, $p \mapsto d$.

Example 2.12. We discuss the quantum deformation of the previous example. Consider the algebra $A_{q}$ that is the algebra $\mathbb{C}_{q}\langle a, b, c, d\rangle$ freely generated (over $\mathbb{C}_{q}=\mathbb{C}\left[q, q^{-1}\right], q$ an indeterminate that may be specialized to a complex number) by the symbols $a, b, c, d$, 
modulo the ideal $I_{M}$ generated by the $q$-commutation relations (or Manin relations, cf. Definition 5.1),

$$
\begin{gathered}
a b=q^{-1} b a, \quad a c=q^{-1} c a, \quad b d=q^{-1} d b, \quad c d=q^{-1} d c, \\
b c=c b \quad a d-d a=\left(q^{-1}-q\right) b c
\end{gathered}
$$

and modulo the ideal $\left(a d-q^{-1} b c-1\right)$ generated by the determinant relation. In short:

$$
A_{q}:=\mathcal{O}_{q}\left(\mathrm{SL}_{2}\right)=\mathbb{C}_{q}\langle a, b, c, d\rangle / I_{M}+\left(a d-q^{-1} b c-1\right) .
$$

Let us similarly define

$$
\mathcal{O}_{q}(P):=\mathbb{C}_{q}\left\langle t, t^{-1}, p\right\rangle /\left(t p-q^{-1} p t\right):=\mathbb{C}_{q}\langle t, s, p\rangle /\left(t s-1, s t-1, t p-q^{-1} p t\right) .
$$

Let $U_{i}$ be a cover of $M=\mathrm{SL}_{2}(\mathbb{C}) / P$ as in Example 2.11. In analogy with the classical case we define $A_{q 1}:=A_{q}\left[a^{-1}\right], A_{q 2}:=A_{q}\left[c^{-1}\right]$, the noncommutative localizations in the elements $a$ and $c$ respectively. The coinvariants are given by

$$
B_{q 1}=\mathbb{C}_{q}\left[a^{-1} c\right] \simeq \mathbb{C}_{q}[u], \quad B_{q 2}=\mathbb{C}_{q}\left[c^{-1} a\right] \simeq \mathbb{C}_{q}[v] .
$$

The ringed space $\left(\mathbf{P}^{1}(\mathbb{C}), \mathcal{O}_{q} \mathbf{P}^{1}(\mathbb{C})\right.$ can be then easily constructed in analogy with the commutative case:

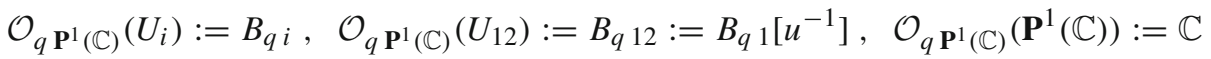

with the nontrivial restriction map given by $r_{q 12,2}: B_{q 2} \rightarrow B_{q 12}, v \mapsto u^{-1}$ that is again well defined since on $U_{12}$ one has $u v=1=v u$.

The natural candidate

$$
\mathcal{F}\left(U_{i}\right):=A_{q i}, \quad \mathcal{F}\left(U_{12}\right):=A_{q 12}:=A_{q 1}\left[c^{-1}\right]=A_{q 2}\left[a^{-1}\right], \quad \mathcal{F}\left(\mathbf{P}^{1}(\mathbb{C})\right)=A_{q},
$$

is again a sheaf of $\mathcal{O}_{q}(P)$-comodule algebras on $\mathbf{P}^{1}(\mathbb{C})$; note in particular that $A_{q 12}$ is well defined since the localization we choose satisfies the Ore condition (see $[39,41]$ ). As in the previous section we define the cleaving maps $j_{i}: \mathcal{O}_{q}(P) \longrightarrow A_{q i}, i=1,2$ on the generators as:

$$
\begin{aligned}
& j_{1}: t^{ \pm 1} \mapsto a^{ \pm 1}, \quad p \mapsto b, \\
& j_{2}: t^{ \pm 1} \mapsto c^{ \pm 1}, \quad p \mapsto d .
\end{aligned}
$$

We observe that $j_{1}$ extends to an algebra map to all $A_{q 1}$ :

$$
j_{1}\left(t p-q^{-1} p t\right)=j_{1}(t) j_{1}(p)-q^{-1} j_{1}(p) j_{1}(t)=a b-q^{-1} b a
$$

and similarly for $j_{2}$. The comodule property of $j_{1}$ (and similarly for $j_{2}$ ) is then easily checked on the generators:

$$
\delta \circ j_{1}(t)=a \otimes t=\left(j_{1} \otimes i d\right) \circ \Delta(t)
$$

and

$$
\delta \circ j_{1}(p)=b \otimes t^{-1}+a \otimes p=\left(j_{1} \otimes i d\right) \circ \Delta(p) .
$$

We can then conclude that $A_{q i}$ are trivial $\mathcal{O}_{q}(P)$-extensions of $B_{q} i$. 
We will study a generalization of the above example in Sect. 5. In that more general setting we will use the following proposition (see e.g. $[14, \S 1.1]$ ),

Proposition 2.13. Let $\mathcal{B}$ be a basis for a topology $\mathcal{T}$ on $M$. Then a $\mathcal{B}$-sheaf of $H$ comodule algebras $\mathcal{F}$ (that is a sheaf defined for the open sets in $\mathcal{B}$ with gluing conditions) extends to a unique sheaf of $H$-comodule algebras on $M$.

Remark 2.14. In Example 2.12, with $\left\{U_{i}\right\}$ open cover of $\mathbf{P}^{1}(\mathbb{C})$, the $\mathcal{B}$-sheaf is the restriction of $\mathcal{F}$ to $\mathcal{B}=\left\{\emptyset, U_{12}, U_{1}, U_{2}\right\}$, and $\mathcal{F}\left(\mathbf{P}^{1}(\mathbb{C})\right)$ is recovered as the pullback $\mathcal{F}\left(\mathbf{P}^{1}(\mathbb{C})\right)=\left\{(f, g) \in \mathcal{F}\left(U_{1}\right) \times \mathcal{F}\left(U_{2}\right) ; r_{q 12,1}(f)=r_{q 12,2}(g)\right\}$ of $\mathcal{O}_{q}(P)$-comodule algebras (here $r_{q 12, i}: A_{q i} \rightarrow A_{q} 12$ are the obvious restriction maps).

\section{Quantum Homogenous Projective Varieties}

A homogenous projective variety can be realized as quotient of affine algebraic groups $G, P$. Its homogenous coordinate $\operatorname{ring} \tilde{\mathcal{O}}(G / P)$ with respect to a chosen projective embedding, when corresponding to a very ample line bundle $\mathcal{L}$, is obtained via a section of $\mathcal{L}$; this is a given element $t \in \mathcal{O}(G)$. A quantum homogenous projective variety $\tilde{\mathcal{O}}_{q}(G / P)$ can be similarly characterized via a quantum section $d \in \mathcal{O}_{q}(G)$. We review this construction due to [10], see also [17], adapting, for the reader's convenience, the main definitions and results to the present setting that differs from the first reference setting (there the accent was on Poisson geometry and Quantum Duality principle).

3.1. Projective embeddings of homogeneous spaces. If $G$ is a semisimple algebraic group, $P$ a parabolic subgroup, the quotient $G / P$ is a projective variety and the projection $G \longrightarrow G / P$ is a principal bundle (see Definition 2.1). $G / P$ is an homogeneous space for the $G$-action and just an homogeneous variety for the $P$-action, which is not transitive.

We now recall how a character of $P$ determines a projective embedding of $G / P$ and its coordinate ring $\tilde{\mathcal{O}}(G / P)$. Given a representation $\rho$ of $P$ on some vector space $V$, we can construct a vector bundle associated to it, namely

$$
\mathcal{V}:=G \times{ }_{P} V=G \times V / \simeq, \quad(g p, v) \simeq\left(g, \rho(p)^{-1} v\right), \quad \forall p \in P, g \in G, v \in V .
$$

The space of global sections of this bundle is identified with the induced module (see, e.g., [25] for more details)

$$
H^{0}(G / P, \mathcal{V})=\left\{f: G \rightarrow V \mid f \text { is regular, } f(g p)=\rho(p)^{-1} f(g)\right\} .
$$

In particular, for $\chi: P \longrightarrow k^{*}$ a character of $P$, i.e. a one dimensional representation of $P$ on $L \simeq k$, we can consider $\mathcal{L}^{n}:=G \times{ }_{P} L^{\otimes n}$ and define

$$
\begin{array}{r}
\tilde{\mathcal{O}}(G / P)_{n}:=H^{0}\left(G / P, \mathcal{L}^{n}\right) \\
\tilde{\mathcal{O}}(G / P):=\bigoplus_{n \geq 0} \tilde{\mathcal{O}}(G / P)_{n} \subset \mathcal{O}(G) .
\end{array}
$$

Assume $\mathcal{L}$ is very ample, i.e. it is generated by a set of global sections $f_{0}, f_{1}, \ldots$, $f_{N} \in \tilde{\mathcal{O}}(G / P)_{1}$; so that the algebra $\tilde{\mathcal{O}}(G / P)$ is graded and generated in degree 1 (by the $f_{i}$ 's). Then $\tilde{\mathcal{O}}(G / P)$ is the homogeneous coordinate ring of the projective variety $G / P$ with respect to the embedding given via the global sections of $\mathcal{L}$ (see [21], p. 176). 
Observation 3.1. While $\mathcal{O}_{G / P}$ denotes the structure sheaf of $G / P$, so that $\mathcal{O}_{G / P}(G / P)$ is the space of global sections, that is $k$ since $G / P$ is a projective variety, $\tilde{\mathcal{O}}(G / P)$ denotes the homogeneous coordinate ring of $G / P$ with respect to a given projective embedding.

We want to reformulate this classical construction in purely Hopf algebraic terms. The character $\chi$ is a group-like element in the coalgebra $\mathcal{O}(P)$. The same holds for all powers $\chi^{n}(n \in \mathbb{N})$. As the $\chi^{n}$ 's are group-like, if they are pairwise different they also are linearly independent, which ensures that the sum $\sum_{n \in \mathbb{N}} \tilde{\mathcal{O}}(G / P)_{n}$, inside $\mathcal{O}(G)$, is a direct one. Moreover, once the embedding is given, each summand $\tilde{\mathcal{O}}(G / P)_{n}$ can be described in purely Hopf algebraic terms as

$$
\begin{aligned}
\tilde{\mathcal{O}}(G / P)_{n} & :=\left\{f \in \mathcal{O}(G) \mid f(g p)=\chi^{n}\left(p^{-1}\right) f(g)\right\} \\
& =\left\{f \in \mathcal{O}(G) \mid((i d \otimes \pi) \circ \Delta)(f)=f \otimes S\left(\chi^{n}\right)\right\}
\end{aligned}
$$

with $\pi: \mathcal{O}(G) \rightarrow \mathcal{O}(P)$ the standard projection, $S$ the antipode of $\mathcal{O}(P)$. Lifting $S(\chi) \in \mathcal{O}(P)$ to an element $t \in \mathcal{O}(G)$ we have the following proposition.

Proposition 3.2. Let $P$ be a parabolic subgroup of a semisimple algebraic group $G$ and denote by $\pi: \mathcal{O}(G) \longrightarrow \mathcal{O}(P)$ the natural projection dual to the inclusion $P \subset G$. If $G / P$ is embedded into some projective space via some very ample line bundle $\mathcal{L}$ then there exists an element $t \in \mathcal{O}(G)$ such that

$$
\begin{aligned}
& \Delta_{\pi}(t):=((i d \otimes \pi) \circ \Delta)(t)=t \otimes \pi(t) \\
& \pi\left(t^{m}\right) \neq \pi\left(t^{n}\right) \quad \forall m \neq n \in \mathbb{N} \\
& \tilde{\mathcal{O}}(G / P)_{n}=\left\{f \in \mathcal{O}(G) \mid(i d \otimes \pi) \Delta(f)=f \otimes \pi\left(t^{n}\right)\right\} \\
& \tilde{\mathcal{O}}(G / P)=\bigoplus_{n \in \mathbb{N}} \tilde{\mathcal{O}}(G / P)_{n}
\end{aligned}
$$

where $\tilde{\mathcal{O}}(G / P)$ is the homogeneous coordinate ring generated by the global sections of $\mathcal{L}$, i.e. generated by $\tilde{\mathcal{O}}(G / P)_{1}$.

Vice-versa, given $t \in \mathcal{O}(G)$ satisfying (5), (6), if $\tilde{\mathcal{O}}(G / P)$ as defined in (7), (8) is generated in degree 1 , namely by $\tilde{\mathcal{O}}(G / P)_{1}$, then $\tilde{\mathcal{O}}(G / P)$ is the homogeneous coordinate ring of the projective variety $G / P$ associated with the projective embedding of $G / P$ given by the very ample line bundle $\mathcal{L}=G \times{ }_{P} k$, the $P$-action on the ground field $k$ being induced by $\pi(t)$.

Proof. See [10].

Notice that while $S(\chi)=\pi(t)$ is group-like, $t$ has an "almost group-like property", given by (5). We call an element $t \in \mathcal{O}(G)$ satisfying (5), (6) a classical section because $t \in \tilde{\mathcal{O}}(G / P)_{1}$. The line bundle $\mathcal{L}$ and the homogenous coordinate ring $\tilde{\mathcal{O}}(G / P)$ depend only on $\pi(t)$, not on the lift $t$.

Remark 3.3. We point out that $\tilde{\mathcal{O}}(G / P)$ is a unital subalgebra as well as a (left) coideal of $\mathcal{O}(G)$; the latter property reflects the fact that $G / P$ is a (left) $G$-space. Thus, the restriction of the comultiplication of $\mathcal{O}(G)$, namely

$$
\left.\Delta\right|_{\tilde{\mathcal{O}}(G / P)}: \tilde{\mathcal{O}}(G / P) \longrightarrow \mathcal{O}(G) \otimes \tilde{\mathcal{O}}(G / P)
$$


is a left coaction of $\mathcal{O}(G)$ on $\tilde{\mathcal{O}}(G / P)$, which structures $\tilde{\mathcal{O}}(G / P)$ into an $\mathcal{O}(G)$ comodule algebra. Moreover $\tilde{\mathcal{O}}(G / P)$ is graded and the coaction $\left.\Delta\right|_{\tilde{\mathcal{O}}(G / P)}$ is also graded with respect to the trivial grading on $\mathcal{O}(G)$, so that each $\tilde{\mathcal{O}}(G / P)_{n}$ is indeed a coideal of $\mathcal{O}(G)$ as well.

3.2. Quantum homogeneous projective varieties and quantum sections. We quickly recall some definitions of quantum deformations and quantum groups, establishing our notation. We define quantum homogeneous spaces and then turn to the quantization of the picture described in the previous section.

Definition 3.4. By quantization of $\mathcal{O}(G)$, we mean a Hopf algebra $\mathcal{O}_{q}(G)$ over the ground ring $k_{q}:=k\left[q, q^{-1}\right]$, where $q$ is an indeterminate, such that:

1. the specialization of $\mathcal{O}_{q}(G)$ at $q=1$, that is $\mathcal{O}_{q}(G) /(q-1) \mathcal{O}_{q}(G)$, is isomorphic to $\mathcal{O}(G)$ as a Hopf algebra;

2. $\mathcal{O}_{q}(G)$ is torsion-free, as a $k_{q}$-module;

We also call $\mathcal{O}_{q}(G)$ a quantum deformation of $G$, or for short, quantum group.

We also say that the $k_{q}$-algebra $\mathcal{O}_{q}(M)$ is a quantization of $\mathcal{O}(M)$ if it is torsion-free and $\mathcal{O}_{q}(M) /(q-1) \mathcal{O}_{q}(M) \simeq \mathcal{O}(M)$. If $\mathcal{O}(M)$ is the coordinate ring of an affine variety $M$, we further say that $\mathcal{O}_{q}(M)$ is a quantization of $M$. If $\tilde{\mathcal{O}}(M)$ is the homogeneous coordinate ring of a projective variety, with respect to a given projective embedding, we say that $\tilde{\mathcal{O}}_{q}(M)$ is a quantization of $M$ provided it is graded and the quantization preserves the homogeneous components.

We next define quantum homogeneous varieties, in this case $M=G / P$.

Definition 3.5. Let $G / P$ be a homogeneous space with respect to the action of an algebraic group $G$. If $G / P$ is affine we say that its quantization $\mathcal{O}_{q}(G / P)$ is a quantum homogeneous variety (space) if $\mathcal{O}_{q}(G / P)$ is a subalgebra of $\mathcal{O}_{q}(G)$ and an $\mathcal{O}_{q}(G)$ comodule algebra. If $G / P$ is projective and $\tilde{\mathcal{O}}(G / P)$ is its homogeneous coordinate ring with respect to a given projective embedding, then we ask its quantization $\tilde{\mathcal{O}}_{q}(G / P)$ to be a $\mathcal{O}_{q}(G)$-comodule subalgebra of $\mathcal{O}_{q}(G)$. We furtherly ask the algebra $\tilde{\mathcal{O}}_{q}(G / P)$ to be graded and the $\mathcal{O}_{q}(G)$-coaction to preserve the grading. In this case we call $\tilde{\mathcal{O}}_{q}(G / P)$ a quantum homogeneous projective variety.

Let $\mathcal{O}_{q}(G)$ be a quantum group and $\mathcal{O}_{q}(P)$ a quantum subgroup (quotient Hopf algebra), quantizations respectively of $G$ and $P$ as above. Since from Proposition 3.2 a classical section $t$ defines a line bundle on $G / P$ and a projective embedding, we study a quantum projective embedding by quantizing this classical section.

Definition 3.6. A quantum section of the line bundle $\mathcal{L}$ on $G / P$ associated with the classical section $t$, is an element $d \in \mathcal{O}_{q}(G)$ such that

1. $(i d \otimes \pi) \Delta(d)=d \otimes \pi(d)$, i.e. $\Delta(d)-d \otimes d \in \mathcal{O}_{q}(G) \otimes I_{q}(P)$

2. $d \equiv t, \bmod (q-1)$

where $\pi: \mathcal{O}_{q}(G) \longrightarrow \mathcal{O}_{q}(P):=\mathcal{O}_{q}(G) / I_{q}(P), I_{q}(P) \subset \mathcal{O}_{q}(G)$ being a Hopf ideal, quantization of the Hopf ideal $I(P)$ defining $P$. 
Define now:

$$
\begin{aligned}
\tilde{\mathcal{O}}_{q}(G / P) & :=\sum_{n \in \mathbb{N}} \tilde{\mathcal{O}}_{q}(G / P)_{n}, \quad \text { where } \\
\tilde{\mathcal{O}}_{q}(G / P)_{n} & :=\left\{f \in \mathcal{O}_{q}(G) \mid(i d \otimes \pi) \Delta(f)=f \otimes \pi\left(d^{n}\right)\right\} .
\end{aligned}
$$

We recall a result from [10].

Theorem 3.7. Let $d$ be a quantum section on $G / P$. Then

1. $\tilde{\mathcal{O}}_{q}(G / P)$ is a graded algebra,

$$
\tilde{\mathcal{O}}_{q}(G / P)_{r} \cdot \tilde{\mathcal{O}}_{q}(G / P)_{s} \subset \tilde{\mathcal{O}}_{q}(G / P)_{r+s}, \quad \tilde{\mathcal{O}}_{q}(G / P)=\bigoplus_{n \in \mathbb{N}} \tilde{\mathcal{O}}_{q}(G / P)_{n}
$$

2. $\tilde{\mathcal{O}}_{q}(G / P)$ is a graded $\tilde{\mathcal{O}}_{q}(G)$-comodule algebra, via the restriction of the comultiplication $\Delta$ in $\mathcal{O}_{q}(G)$,

$$
\left.\Delta\right|_{\tilde{\mathcal{O}}_{q}(G / P)}: \tilde{\mathcal{O}}_{q}(G / P) \longrightarrow \mathcal{O}_{q}(G) \otimes \tilde{\mathcal{O}}_{q}(G / P)
$$

where we consider $\mathcal{O}_{q}(G)$ with the trivial grading.

3. As algebra $\tilde{\mathcal{O}}_{q}(G / P)$ is a subalgebra of $\mathcal{O}_{q}(G)$.

Hence $\tilde{\mathcal{O}}_{q}(G / P)$ is a quantum homogeneous projective variety.

From now on we assume that $\tilde{\mathcal{O}}_{q}(G / P)$ is generated in degree one, namely by $\tilde{\mathcal{O}}_{q}(G / P)_{1}$. The quantum Grassmannian and flag are examples of this construction and they are both generated in degree one.

Example 3.8. Let us consider the case $G=\mathrm{SL}_{n}(\mathbb{C})$ and $P$ the maximal parabolic subgroup of $G$ :

$$
P=\left\{\left(\begin{array}{cc}
t_{r \times r} & p_{r \times n-r} \\
0_{n-r \times r} & s_{n-r \times n-r}
\end{array}\right)\right\} \subset \mathrm{SL}_{n}(\mathbb{C}) .
$$

The quotient $G / P$ is the Grassmannian Gr of $r$ spaces into the $n$ dimensional vector space $\mathbb{C}^{n}$. It is a projective variety and it can be embedded, via the Plücker embedding, into the projective space $\mathbf{P}^{N}(\mathbb{C})$ where $N=\left(\begin{array}{l}n \\ r\end{array}\right)$. This embedding corresponds to the character:

$$
P \ni\left(\begin{array}{ll}
t & p \\
0 & s
\end{array}\right) \mapsto \operatorname{det}(t) \in \mathbb{C}^{\times} .
$$

The coordinate ring $\mathcal{O}(\mathrm{Gr})$ of $\mathrm{Gr}$, with respect to the Plücker embedding, is realized as the graded subring of $\mathcal{O}\left(\mathrm{SL}_{n}\right)$ generated by the determinants $d_{I}$ of the minors obtained by taking (distinct) rows $I=\left(i_{1}, \ldots, i_{r}\right)$ and columns $1, \ldots, r$. In fact one can readily check that $d=\operatorname{det}\left(a_{i j}\right)_{1 \leq i, j \leq r}$ is a classical section and, denoting by $\pi: \mathcal{O}\left(\mathrm{SL}_{n}\right) \longrightarrow$ $\mathcal{O}(P)$ the natural projection dual to the inclusion $P \subset \mathrm{SL}_{n}$, that

$$
(i d \otimes \pi) \Delta\left(d_{I}\right)=d_{I} \otimes \pi(d) .
$$

In [15] the quantum Grassmannian $\mathcal{O}_{q}(\mathrm{Gr})$ is defined as the graded subring of $\mathcal{O}_{q}\left(\mathrm{SL}_{n}\right)$ generated by all of the quantum determinants $D_{I}$ of the minors obtained 
by taking (distinct) rows $I=\left(i_{1}, \ldots, i_{r}\right)$ and columns $1, \ldots, r$. It is a quantum deformation of $\mathcal{O}(\mathrm{Gr})$ and a quantum homogeneous projective space for the quantum group $\mathcal{O}_{q}\left(\mathrm{SL}_{n}\right)$, (see $[15,17]$ for more details). Again one can readily check that $d=D_{1 \ldots r}$ is a quantum section and that

$$
(i d \otimes \pi) \Delta\left(D_{I}\right)=D_{I} \otimes \pi(d),
$$

where $\mathcal{O}_{q}(P)=\mathcal{O}_{q}(G) / I_{q}(P)$ is the quantum subgroup of $\mathcal{O}_{q}(G)$ defined by the Hopf ideal $I_{q}(P)=\left(a_{i j}\right)$ generated by the elements $a_{i j}$ for $r+1 \leq i \leq n$ and $1 \leq j \leq r$, and $\pi: \mathcal{O}_{q}(G) \longrightarrow \mathcal{O}_{q}(P)$.

\section{Quantum Principal Bundles from Parabolic Quotients $G / P$}

In the previous section we have seen how to construct a quantum homogenous projective variety $\tilde{\mathcal{O}}_{q}(G / P)$ given a quantum section $d \in \mathcal{O}_{q}(G)$. We here show how quantum sections lead to quantum principal bundles over quantum homogeneous projective varieties.

4.1. Sheaves of comodule algebras. Let as before $G$ be a semisimple algebraic group, $P$ a parabolic subgroup.

We start with a classical observation recalling the construction of a (finite) basis $\left\{t_{i}\right\}_{i \in \mathcal{I}}$ of the module of global sections of the very ample line bundle $\mathcal{L} \rightarrow G / P$ associated with a classical section $t \in \mathcal{O}(G)$. We also construct the corresponding open cover $\left\{V_{i}\right\}_{i \in \mathcal{I}}$ of $G$.

Observation 4.1. Recalling Proposition 3.2, we consider an element in $t \in \mathcal{O}(G)$ satisfying (5) and (6) and defining a very ample line bundle $\mathcal{L} \rightarrow G / P$, with $t \in \tilde{\mathcal{O}}(G / P)_{1} \subset$ $\mathcal{O}(G)$ that is now a section of $\mathcal{L}$. Let $\Delta(t)=\sum t_{(1)} \otimes t_{(2)}=\sum_{i \in \mathcal{I}} t^{i} \otimes t_{i}$ be its coproduct and notice that the elements $t_{i}$ can be chosen to be linearly independent. We now show that $\left\{t_{i}\right\}_{i \in \mathcal{I}}$ is a basis of $\tilde{\mathcal{O}}(G / P)_{1}$, the module of global section of $\mathcal{L}$, hence the $t_{i}$ 's generate $\tilde{\mathcal{O}}(G / P)$ as a (graded) algebra. Indeed, by the Borel-Weyl-Bott theorem, $\tilde{\mathcal{O}}(G / P)_{1}$ is an irreducible $G$ module (corresponding to the infinitesimal weight uniquely associated to $\chi$ ). By the very definition of $\Delta$, the $G$-action on $t$ is given by, for all $g, x \in G$ :

$$
(g \cdot t)(x)=t\left(g^{-1} x\right)=\Delta(t)\left(g^{-1} \otimes x\right)=\sum t^{i}\left(g^{-1}\right) t_{i}(x) .
$$

Since $\tilde{\mathcal{O}}(G / P)_{1}$ is irreducible, for any $f \in \tilde{\mathcal{O}}(G / P)_{1}$ there exists a $g \in G$, such that $f=g \cdot t$ and consequently $f$ is a linear combination of the $t_{i}$ 's by (10). Hence the $t_{i}$ 's form a basis of $\tilde{\mathcal{O}}(G / P)_{1}$.

Furthermore, a covering of $G$ is given by $\left\{V_{i}\right\}_{i \in \mathcal{I}}$, where the open sets $V_{i}$ are defined by the non vanishing of the corresponding $t_{i} \in \mathcal{O}(G)$. This is so because the line bundle $\mathcal{L}$ defines a projective embedding of $G / P$, hence there are no common zeros for its global sections.

Based on the previous observation we have the following important property of the quantum homogeneous projective variety $\tilde{\mathcal{O}}_{q}(G / P)$. 
Lemma 4.2. Let $d$ be a quantum section, and $\Delta(d)=\sum d_{(1)} \otimes d_{(2)}=\sum_{i \in \mathcal{I}} d^{i} \otimes d_{i}$ be its coproduct. Then the $d_{i}$ 's can be chosen so to form a basis of $\tilde{\mathcal{O}}_{q}(G / P)_{1}$ as $k_{q}$ free module, hence of $\tilde{\mathcal{O}}_{q}(G / P)$ as graded algebra.

Proof. The fact that the $d_{i}$ 's belong to $\tilde{\mathcal{O}}_{q}(G / P)_{1}$ is non trivial, but it is an immediate consequence of Proposition 3.10 in [10]. The property that they generate $\tilde{\mathcal{O}}_{q}(G / P)_{1}$ as $k_{q}$ free module is a consequence of the same property being true in the classical setting (see Observation 4.1) and comes through the application of Proposition 1.1 in [20] followed by Lemma 3.10 in [18]. The last property immediately follows from the assumption that $\tilde{\mathcal{O}}_{q}(G / P)$ is generated by $\tilde{\mathcal{O}}_{q}(G / P)_{1}$.

We assume that

$$
S_{i}:=\left\{d_{i}^{r}, r \in \mathbb{Z}_{\geq 0}\right\}, \quad i \in \mathcal{I}
$$

is Ore in order to consider localizations of $\mathcal{O}_{q}(G)$ and hence define a sheaf. We furtherly assume that $S_{i}$ is Ore in the graded subalgebra $\mathcal{O}_{q}(G / P)$ of $\mathcal{O}_{q}(G)$. We can then define:

$$
\mathcal{O}_{q}\left(V_{i}\right):=\mathcal{O}_{q}(G) S_{i}^{-1},
$$

the Ore extension of $\mathcal{O}_{q}(G)$ with respect to the multiplicatively closed set $S_{i}$. Notice that $\mathcal{O}_{q}\left(V_{i}\right)$ is a quantization of $\mathcal{O}\left(V_{i}\right)$, the coordinate ring of the open set $V_{i} \subset G$.

Proposition 4.3. For any $i \in \mathcal{I}$, the algebra $\mathcal{O}_{q}\left(V_{i}\right)$ is an $\mathcal{O}_{q}(P)$-comodule algebra with coaction $\delta_{i}: \mathcal{O}_{q}\left(V_{i}\right) \longrightarrow \mathcal{O}_{q}\left(V_{i}\right) \otimes \mathcal{O}_{q}(P)$ given by:

$$
\delta_{i}(x)=((i d \otimes \pi) \circ \Delta)(x), \quad \delta_{i}\left(d_{i}^{-1}\right)=d_{i}^{-1} \otimes \pi(d)^{-1}, \quad x \in \mathcal{O}_{q}(G)
$$

where with an abuse of notation we write $\pi(d)^{-1}$ for the antipode of $\pi(d)$ in $\mathcal{O}_{q}(P)$.

Proof. Notice that $\mathcal{O}_{q}(G)$ is an $\mathcal{O}_{q}(P)$-comodule algebra with coaction $\Delta_{\pi}=(\mathrm{id} \otimes$ $\pi) \circ \Delta$. Since $\Delta_{\pi}\left(d_{i}\right)=d_{i} \otimes \pi(d)$ is invertible in $\mathcal{O}_{q}\left(V_{i}\right) \otimes \mathcal{O}_{q}(P)$ by the universality of the Ore construction we have our definition of $\delta_{i}$.

Assume now we can form iterated Ore extensions:

$$
\mathcal{O}_{q}\left(V_{i_{1}} \cap \cdots \cap V_{i_{s}}\right):=\mathcal{O}_{q}\left(\cap_{i \in I} V_{i}\right):=\mathcal{O}_{q}(G) S_{i_{1}}^{-1} \ldots S_{i_{s}}^{-1}, \quad I=\left\{i_{1}, \ldots, i_{s}\right\},
$$

with $i_{1}, \ldots i_{s}, s \in \mathcal{I}$, independently from the order, i.e. we assume to have a natural isomorphism between $\mathcal{O}_{q}(G) S_{i}^{-1} S_{j}^{-1}$ and $\mathcal{O}_{q}(G) S_{j}^{-1} S_{i}^{-1}$ and, iteratively, between $\mathcal{O}_{q}(G) S_{i_{1}}^{-1} \ldots S_{i_{s}}^{-1}$ and $\mathcal{O}_{q}(G) S_{i_{\sigma_{1}}}^{-1} \ldots S_{i_{\sigma_{s}}}^{-1}$, with $\sigma$ any permutation of $s$ elements. This is in general a very restrictive hypothesis, neverthless we will see it is verified in some interesting examples in the next section.

We also define:

$$
r_{I J}: \mathcal{O}_{q}\left(\cap_{i \in I} V_{i}\right) \longrightarrow \mathcal{O}_{q}\left(\cap_{j \in J} V_{j}\right), \quad I \subset J
$$

as the natural morphism obtained from the Ore extension.

Setting as usual $V_{I}=\cap_{i \in I} V_{i}$ we immediately have the following proposition (cf. Proposition 4.3). 
Proposition 4.4. $\mathcal{O}_{q}\left(V_{I}\right)$ is an $\mathcal{O}_{q}(P)$-right comodule algebra and the morphisms $r_{I J}$ are $\mathcal{O}_{q}(P)$-right comodule algebra morphisms.

Let us now consider the open sets $U_{I}:=\wp\left(V_{I}\right)$, obtained via the projection $\wp$ : $G \longrightarrow G / P$. We have the following.

Proposition 4.5. The assignment:

$$
U_{I} \mapsto \mathcal{F}\left(U_{I}\right):=\mathcal{O}_{q}\left(V_{I}\right),
$$

with the restriction maps $r_{I J}: \mathcal{O}_{q}\left(V_{I}\right) \rightarrow \mathcal{O}_{q}\left(V_{J}\right)$, defines a sheaf of $\mathcal{O}_{q}(P)$-comodule algebras on $G / P=\cup_{i \in \mathcal{I}} U_{i}$, and more in general on $M:=\cup_{i \in \mathcal{J}} U_{i} \subset G / P$, where $I \subset \mathcal{I}$ and $I \subset \mathcal{J} \subset \mathcal{I}$, respectively.

Proof. The open sets $U_{I}$ with $I \subset \mathcal{I}$ (and the empty set) form a basis $\mathcal{B}$ for a topo$\operatorname{logy}$ on $G / P$. Recalling Proposition 2.13 we just have to show that the assignment $U_{I} \mapsto \mathcal{F}\left(U_{I}\right):=\mathcal{O}_{q}\left(V_{I}\right)$, with the restriction maps $r_{I J}$, defines a $\mathcal{B}$-sheaf of $\mathcal{O}_{q}(P)$ comodule algebras. Since restrictions morphisms are actually algebra inclusions, using the existence of iterated Ore extension and their compatibility this is straighforwardly seen to be a $\mathcal{B}$-sheaf of algebras and of $O_{q}(P)$-comodule algebras.

The sheaf on the more general open submanifold $M=\cup_{i \in \mathcal{J}} U_{i}$ is simply obtained by considering the open sets $U_{I}$ with $I \subset \mathcal{J} \subset \mathcal{I}$.

4.2. Quantum principal bundles on quantum homogeneous spaces. In the previous section we have constructed a sheaf of comodule algebras $\mathcal{F}$ on $M \subset G / P$. We now want to define a quantum ringed space structure on the topological space $M$ as in Definition 2.8 and show that $\mathcal{F}$ is a quantum principal bundle on it. Notice that $M$ coincides with $G / P$ if $\mathcal{J}=\mathcal{I}$, while for $\mathcal{J} \varsubsetneqq \mathcal{I}$, i.e. for a proper subset of the set of indices $\mathcal{I}$ of the open cover $\left\{V_{i}\right\}_{i \in \mathcal{I}}$ of $G$, we have that $M$ is a proper open subset of $G / P$.

By Observation 4.1 we know that $\left\{U_{i}:=\wp\left(V_{i}\right)\right\}_{i \in \mathcal{I}}$ is an open cover of $G / P$. Define $\mathcal{O}_{q}\left(U_{i}\right)$ as the subalgebra of $\mathcal{O}_{q}(G) S_{i}^{-1}$ generated by the elements $d_{k} d_{i}^{-1}$, for $k \in \mathcal{I}$ :

$$
\mathcal{O}_{q}\left(U_{i}\right):=k_{q}\left[d_{k} d_{i}^{-1}\right]_{k \in \mathcal{I}} \subset \mathcal{O}_{q}(G) S_{i}^{-1} .
$$

Because of our (graded) Ore hypothesis, this is also the subalgebra of elements of degree zero inside $\tilde{\mathcal{O}}_{q}(G / P) S_{i}^{-1}$ and, for this reason, it is called the (noncommutative) projective localization of $\tilde{\mathcal{O}}_{q}(G / P)$ at $S_{i}$.

Proposition 4.6. Let the notation be as above. The assignment

$$
U_{I} \mapsto \mathcal{O}_{q}\left(U_{I}\right)
$$

defines a sheaf $\mathcal{O}_{M}$ on $M=\cup_{i \in \mathcal{J}} U_{i}$, hence $\left(M, \mathcal{O}_{M}\right)$ is a quantum ringed space.

Proof. According to Proposition 2.13 it is enough to check that our assignment is a $\mathcal{B}$-sheaf for the basis associated with the open sets $\left\{U_{i}\right\}$, but this is immediate by our hypothesis on the existence of iterated Ore extension and their compatibility.

Proposition 4.7. Let the notation be as above. Then $\mathcal{F}\left(U_{i}\right)^{\operatorname{co} \mathcal{O}_{q}(P)}=\mathcal{O}_{M}\left(U_{i}\right)$, i.e. it is the subring in $\mathcal{F}\left(U_{i}\right)$ generated by the elements $d_{j} d_{i}^{-1}$. 
Proof. By our definition of coaction $\delta_{i}$ (see (12))

$$
\delta_{i}\left(d_{j} d_{i}^{-1}\right)=\left(d_{j} \otimes \pi(d)\right)\left(d_{i}^{-1} \otimes \pi(d)^{-1}\right)=d_{j} d_{i}^{-1} \otimes 1 .
$$

We now need to prove that the $d_{j} d_{i}^{-1}$ generate the subring of coinvariants. Assume $z \in \mathcal{F}\left(U_{i}\right)^{\operatorname{co} \mathcal{O}_{q}(P)} \subset \mathcal{F}\left(U_{i}\right)$. Since $\mathcal{F}\left(U_{i}\right):=\mathcal{O}_{q}(G)\left[S_{i}^{-1}\right]$, then $z d_{i}^{r} \in \mathcal{O}_{q}(G)$ for a suitable $r$. Notice that:

$$
\delta_{i}\left(z d_{i}^{r}\right)=(z \otimes 1)\left(d_{i}^{r} \otimes \pi(d)^{r}\right)=z d_{i}^{r} \otimes \pi(d)^{r} .
$$

Hence $z d_{i}^{r} \in \tilde{\mathcal{O}}_{q}(G / P)_{r}$, which, by Lemma 4.2, is generated by the $d_{j}$ 's:

$$
z d_{i}^{r}=\sum_{\lambda_{j_{i} \ldots j_{r}} \in k_{q}} \lambda_{j_{i} \ldots j_{r}} d_{j_{1}} \ldots d_{j_{r}}
$$

Therefore we have:

$$
z=\sum_{\lambda_{j_{i} \ldots j_{r}} \in k_{q}} \lambda_{j_{i} \ldots j_{r}} d_{j_{1}} \ldots d_{j_{r}} d_{i}^{-r}
$$

We now proceed by induction on $r$. The case $r=0$ is clear. For generic $r$, since $d_{i}$ satisfies the Ore condition:

$$
d_{j_{r}} d_{i}^{-(r-1)}=d_{i}^{-(r-1)} \sum_{\mu_{j_{r} s} \in k_{q}} \mu_{j_{r} s} d_{s}
$$

hence:

$$
z=\sum_{\lambda_{j_{i} \ldots j_{r} \in k_{q}}} \lambda_{j_{i} \ldots j_{r}} d_{j_{1}} \ldots d_{j_{r-1}} d_{i}^{-(r-1)} \sum_{\mu_{j_{r} s} \in k_{q}} \mu_{j_{r} s} d_{s} d_{i}^{-1}
$$

By induction we obtain:

$$
z=\sum_{v_{j_{i} \ldots j_{r} \in k_{q}}} v_{j_{i} \ldots j_{r}} d_{j_{1}} d_{i}^{-1} \ldots d_{j_{r-1}} d_{i}^{-1} \sum_{\mu_{j_{r} s} \in k_{q}} \mu_{j_{r} s} d_{s} d_{i}^{-1}
$$

hence our result.

We conclude summarizing the main results we have obtained.

Theorem 4.8. Let $G$ be a semisimple algebraic group and $P$ a parabolic subgroup, let the quantum group $\mathcal{O}_{q}(G)$ and the quantum subgroup $\mathcal{O}_{q}(P):=\mathcal{O}_{q}(G) / I_{q}(P)$ be the quantizations of the coordinate rings $\mathcal{O}(G)$ and $\mathcal{O}(P)$. Let $d$ be a quantum section (see Definition 3.6), denote with $\left\{d_{i}\right\}_{i \in \mathcal{I}}$ a choice of linearly independent elements in the coproduct $\Delta(d)=\sum_{i \in \mathcal{I}} d^{i} \otimes d_{i}$, and assume they generate the homogenous coordinate ring $\tilde{\mathcal{O}}_{q}(G / P)$ (see Lemma 4.2). Assume furtherly that $\mathcal{O}_{q}\left(V_{i}\right):=\mathcal{O}_{q}(G) S_{i}^{-1}, S_{i}=$ $\left\{d_{i}^{r}, r \in \mathbb{Z}_{\geq 0}\right\}$ is Ore and that subsequent localizations do not depend on the order (see (13)). Then:

1. Let $\mathcal{O}_{q}\left(U_{i}\right):=k_{q}\left[d_{k} d_{i}^{-1}\right]_{k \in \mathcal{I}} \subset \mathcal{O}_{q}(G) S_{i}^{-1}$. The assignment $U_{i} \mapsto \mathcal{O}_{q}\left(U_{i}\right)$ defines a sheaf $\mathcal{O}_{M}$ on $M=\cup_{i \in \mathcal{J}} U_{i}, \mathcal{J} \subset \mathcal{I}$, hence $\left(M, \mathcal{O}_{M}\right)$ is a quantum ringed space. In particular, for $M=G / P(\mathcal{J}=\mathcal{I})$, the sheaf $\mathcal{O}_{G / P}$ is the projective localization of the homogeneous coordinate ring $\tilde{\mathcal{O}}_{q}(G / P)$. 
2. The assignment: $U_{I} \mapsto \mathcal{F}\left(U_{I}\right):=\mathcal{O}_{q}\left(V_{I}\right)$ defines a sheaf $\mathcal{F}$ of $\mathcal{O}_{q}(P)$-comodule algebras on the quantum ringed space $M=\cup_{i \in \mathcal{J}} U_{i} \subset G / P$.

3. $\mathcal{F}^{\mathrm{co} \mathcal{O}_{q}(P)}=\mathcal{O}_{M}$, i.e., the subsheaf $\mathcal{F}^{\operatorname{co} \mathcal{O}_{q}(P)}: U \rightarrow \mathcal{F}(U)^{\operatorname{co} \mathcal{O}_{q}(P)} \subset \mathcal{F}(U)$ is canonically isomorphic to the sheaf $\mathcal{O}_{M}$.

If the sheaf $\mathcal{F}$ is locally principal then $\mathcal{F}$ is a quantum principal bundle, if the sheaf is locally cleft then $\mathcal{F}$ is a locally cleft quantum principal bundle (see Definition 2.10 and Definition 2.9).

Proof. (1) is Proposition 4.6. (2) is Proposition 4.5. (3) is Proposition 4.7.

\section{Examples on Quantum Projective Spaces}

In this section we apply the general theory we have developed and present quantum principal bundles over quantum projective spaces. We hence sharpen the notion of quantum projective space as quantum homogenous space. In this section the ground field is $k=\mathbb{C}$.

5.1. Quantum deformations of function algebras. We start with an important example of quantum group and its quantum homogeneous varieties. For more details see [30] and [15].

Definition 5.1. We define the quantum matrices as the $\mathbb{C}_{q}$ algebra $\mathcal{O}_{q}\left(M_{n}\right)$ :

$$
\mathcal{O}_{q}\left(\mathrm{M}_{n}\right)=\mathbb{C}_{q}\left\langle a_{i j}\right\rangle / I_{\mathrm{M}}
$$

where $i, j=1, \ldots n$ and $I_{\mathrm{M}}$ is the ideal of the Manin relations:

$$
\begin{gathered}
a_{i j} a_{k j}=q^{-1} a_{k j} a_{i j} \quad i<k, \quad a_{i j} a_{k l}=a_{k l} a_{i j} \quad i<k, j>l \text { or } i>k, j<l, \\
a_{i j} a_{i l}=q^{-1} a_{i l} a_{i j} \quad j<l, \quad a_{i j} a_{k l}-a_{k l} a_{i j}=\left(q^{-1}-q\right) a_{i l} a_{k j} \quad i<k, j<l .
\end{gathered}
$$

The quantum matrix algebra $\mathcal{O}_{q}\left(\mathrm{M}_{n}\right)$ is a bialgebra, with comultiplication and counit given by:

$$
\Delta\left(a_{i j}\right)=\sum_{k} a_{i k} \otimes a_{k j}, \quad \varepsilon\left(a_{i j}\right)=\delta_{i j} .
$$

We define the quantum general linear group to be the algebra

$$
\mathcal{O}_{q}\left(\mathrm{GL}_{n}\right)=\mathcal{O}_{q}\left(\mathrm{M}_{n}\right)\left[\operatorname{det}_{q}^{-1}\right]
$$

where $\operatorname{det}_{q}=\operatorname{det}_{q}\left(a_{i j}\right)$ is the quantum determinant:

$$
\operatorname{det}_{q}\left(a_{i j}\right)=\sum_{\sigma}(-q)^{-\ell(\sigma)} a_{1 \sigma(1)} \ldots a_{n \sigma(n)}=\sum_{\sigma}(-q)^{-\ell(\sigma)} a_{\sigma(1) 1} \ldots a_{\sigma(n) n},
$$

here $\ell(\sigma)$ is the length of the permutation $\sigma$ (see [37] for more details on quantum determinants).

We define the quantum special linear group to be the algebra

$$
\mathcal{O}_{q}\left(\mathrm{SL}_{n}\right)=\mathcal{O}_{q}(M) /\left(\operatorname{det}_{q}-1\right)
$$

$\mathcal{O}_{q}\left(\mathrm{GL}_{n}\right)$ and $\mathcal{O}_{q}\left(\mathrm{SL}_{n}\right)$ are Hopf algebras and quantum deformations respectively of the general linear and the special linear groups. 
5.2. Quantum principal bundles on quantum projective spaces. We consider the special case of a maximal parabolic subgroup $P$ of $G=\mathrm{SL}_{n}(\mathbb{C})$ of the form:

$$
P=\left\{\left(\begin{array}{cccc}
p_{11} & p_{12} & \ldots & p_{1 n} \\
0 & p_{22} & \ldots & p_{2 n} \\
\vdots & & \vdots \\
0 & p_{n 2} & \ldots & p_{n n}
\end{array}\right)\right\} \subset G=\left\{A=\left(\begin{array}{ccc}
a_{11} & \ldots & a_{1 n} \\
a_{21} & \ldots & a_{2 n} \\
\vdots & & \vdots \\
a_{n 1} & \ldots & a_{n n}
\end{array}\right), \operatorname{det}(A)=1\right\} .
$$

In this case $G / P \simeq \mathbf{P}^{n-1}(\mathbb{C})$ is the complex projective space, and $\tilde{\mathcal{O}}\left(\mathbf{P}^{n-1}\right)$ is the corresponding free graded ring with $n$ generators. Its quantization $\tilde{\mathcal{O}}_{q}\left(\mathbf{P}^{n-1}\right)$ is well known and, for example, it is constructed in detail in [15] (see Theorem 5.4 for $r=1$ ), see also [12]. $\tilde{\mathcal{O}}_{q}\left(\mathbf{P}^{n-1}\right)$ is the subring of $\mathcal{O}_{q}\left(\mathrm{SL}_{n}\right)$ generated by the elements $x_{i}=a_{i 1}$, $i \in \mathcal{I}=\{1, \ldots n\}$. We can immediately give a presentation:

$$
\tilde{\mathcal{O}}_{q}\left(\mathbf{P}^{n-1}\right)=\mathbb{C}_{q}\left\langle x_{1}, \ldots, x_{n}\right\rangle /\left(x_{i} x_{j}-q^{-1} x_{j} x_{i}, i<j\right) .
$$

We reinterpret this construction within the present framework, first showing that $\tilde{\mathcal{O}}_{q}\left(\mathbf{P}^{n-1}\right)$ is a quantum homogeneous projective space according to Definition 3.5 and then constructing, along Theorem 4.8, an $\mathcal{O}_{q}(P)$-principal bundle on the ringed space obtained via projective localizations of $\tilde{\mathcal{O}}_{q}\left(\mathbf{P}^{n-1}\right)$.

Let $\mathcal{O}_{q}(G)=\mathcal{O}_{q}\left(\mathrm{SL}_{n}\right)$ be the quantum special linear group of Definition 5.1, and define the quantum parabolic subgroup

$$
\mathcal{O}_{q}(P):=\mathcal{O}_{q}\left(\mathrm{SL}_{n}\right) / I_{q}(P),
$$

where $I_{q}(P)=\left(a_{\alpha 1}\right)$ is the Hopf ideal generated by $a_{\alpha 1}, \alpha \in\{2, \ldots n\}$. We use coordinates $p_{i j}$ for the images of the generators $a_{i j}$ under $\pi: \mathcal{O}_{q}\left(\operatorname{SL}_{n}\right) \longrightarrow \mathcal{O}_{q}(P)$. We notice (cf. Example 3.8) that $d=a_{11} \in \mathcal{O}_{q}\left(\mathrm{SL}_{n}\right)$ is a quantum section, in fact

$$
\Delta_{\pi}\left(a_{11}\right)=a_{11} \otimes p_{11}, \quad p_{11}=\pi\left(a_{11}\right) .
$$

Furthermore, from the coproduct $\Delta\left(a_{11}\right)=\sum_{i \in \mathcal{I}} a_{1 i} \otimes a_{i 1}$ we choose the linearly independent elements $d_{i}$ in $\Delta(d)=\sum_{i \in \mathcal{I}} d^{i} \otimes d_{i}$, to be

$$
d_{i}=a_{i 1} \text {. }
$$

Hence, by Lemma 4.2, the elements $d_{i} \operatorname{span} \tilde{\mathcal{O}}_{q}\left(\mathrm{SL}_{n} / P\right)_{1}$, as defined in (9). The quantum homogeneous projective variety $\tilde{\mathcal{O}}_{q}\left(\mathrm{SL}_{n} / P\right)$ is generated in degree one, cf. Example 3.8 , and one can see immediately that $\tilde{\mathcal{O}}_{q}\left(\mathrm{SL}_{n} / P\right)$ coincides with $\tilde{\mathcal{O}}_{q}\left(\mathbf{P}^{n-1}\right)$, as defined in (17).

We now structure $\tilde{\mathcal{O}}_{q}\left(\mathbf{P}^{n-1}\right)$ as a quantum ringed space and construct a sheaf of locally trivial $\mathcal{O}_{q}(P)$-comodule algebras, i.e., a quantum principal bundle on the quantum projective space $\tilde{\mathcal{O}}_{q}\left(\mathbf{P}^{n-1}\right)$, where $\mathcal{O}_{q}(P)$ is the quantum parabolic subgroup of $\mathcal{O}\left(\mathrm{SL}_{n}\right)$ defined in (18).

Let us consider the two classical open covers of the topological spaces $\mathrm{SL}_{n}(\mathbb{C})$ and $\mathbf{P}^{n-1}(\mathbb{C})$ respectively:

$$
\begin{array}{cc}
\mathrm{SL}_{n}(\mathbb{C})=\cup_{i} V_{i}, & V_{i}=\left\{g \in \mathrm{SL}_{n}(\mathbb{C}) \mid a_{i 1}^{0}(g) \neq 0\right\} \\
\mathbf{P}^{n-1}(\mathbb{C})=\cup_{i} U_{i}, & U_{i}=\left\{z \in \mathbf{P}^{n-1}(\mathbb{C}) \mid x_{i}^{0}(z) \neq 0\right\}
\end{array}
$$

where $a_{i j}^{0}$ denote the generators of $\mathcal{O}\left(\mathrm{SL}_{n}\right)$ and similarly $x_{i}^{0}$ those of $\tilde{\mathcal{O}}\left(\mathbf{P}^{n-1}\right), i, j=$ $1, \ldots, n$. Evidently, $\wp\left(V_{i}\right)=U_{i}, \wp: \mathrm{SL}_{n}(\mathbb{C}) \longrightarrow \mathrm{SL}_{n}(\mathbb{C}) / P=\mathbf{P}^{n-1}(\mathbb{C})$. 
Lemma 5.2. The multiplicative set $S_{i}=\left\{a_{i 1}^{k}\right\}_{k \in \mathbb{N}} \subset \mathcal{O}_{q}\left(\mathrm{SL}_{n}\right)$ satisfies the Ore condition. Furthermore, $\mathcal{O}_{q}\left(\mathrm{SL}_{n}\right) S_{i_{1}}^{-1} \ldots S_{i_{s}}^{-1}$, does not depend on the order of the Ore extensions.

Proof. See [40, pp. 4 and 5]. Notice that $a_{i 1}$ is a quantum minor of order 1 and two such minors $q$-commute, hence their product forms an Ore set.

As a corollary of Theorem 4.8 we then immediately obtain

Proposition 5.3. Let the notation be as in the previous section, with $\mathcal{I}=\{1, \ldots n\}$. The assignment:

$$
U_{I} \longmapsto \mathcal{F}\left(U_{I}\right):=\mathcal{O}_{q}\left(V_{I}\right):=\mathcal{O}_{q}\left(\mathrm{SL}_{n}\right) S_{i_{1}}^{-1} \ldots S_{i_{s}}^{-1}, \quad I=\left\{i_{1}, \ldots, i_{s}\right\},
$$

with $i_{1}, \ldots i_{s}, s \in \mathcal{I}$, defines a sheaf of $\mathcal{O}_{q}(P)$-comodule algebras on $\mathrm{SL}_{n}(\mathbb{C}) / P$. Furthermore, $\mathcal{F}\left(U_{i}\right)^{\operatorname{co} \mathcal{O}_{q}(P)}$ is generated by $a_{i 1} a_{11}^{-1}, \mathcal{F}^{\mathrm{co} \mathcal{O}_{q}(P)}$ equals the projective localization of $\tilde{\mathcal{O}}_{q}\left(\mathbf{P}^{n-1}\right)$ and $\left(\mathrm{SL}_{n}(\mathbb{C}) / P, \mathcal{F}^{\operatorname{co} \mathcal{O}_{q}(P)}\right)$ is a quantum ringed space.

We now prove that $\mathcal{F}$ is a locally cleft quantum principal bundle. We actually show the stronger local triviality condition, i.e., the collection of maps $j_{i}: \mathcal{O}_{q}(P) \rightarrow \mathcal{F}\left(U_{i}\right)$ are $\mathcal{O}_{q}(P)$-comodule algebra maps, this implies that they are cleaving maps (cf. Remark 2.5).

We first study the map $j_{1}$. Let $a_{i j} \in \mathcal{F}\left(U_{1}\right):=\mathcal{O}_{q}\left(\mathrm{SL}_{n}\right) S_{1}^{-1}=\mathcal{O}_{q}\left(\mathrm{SL}_{n}\right)\left[a_{11}^{-1}\right]$, $i, j=1, \ldots n$; since $a_{11}$ is invertible we have the matrix factorization

$$
\left(a_{i j}\right)=\left(\begin{array}{cc}
1 & 0 \\
a_{\alpha 1} a_{11}^{-1} & 11
\end{array}\right)\left(\begin{array}{cc}
a_{11} & a_{1 \beta} \\
0 & a_{\alpha \beta}-a_{\alpha 1} a_{11}^{-1} a_{1 \beta}
\end{array}\right)=\left(\begin{array}{cc}
1 & 0 \\
a_{\alpha 1} a_{11}^{-1} & 11
\end{array}\right)\left(\begin{array}{cc}
a_{11} & a_{1 \beta} \\
0 & a_{11}^{-1} D_{1 \alpha}^{1 \beta}
\end{array}\right)
$$

where $\alpha, \beta=2, \ldots n$, and $D_{i j}^{k l}=a_{i k} a_{j l}-q^{-1} a_{i l} a_{j k}$, with $i<j$ and $k<l$, denotes the quantum determinant of the $2 \times 2$ quantum matrix obtained by taking rows $i, j$ and columns $k, l$.

In the commutative case this factorization corresponds to the trivialization $V_{1} \simeq$ $\mathbb{C}^{n-1} \times P$ of the open $V_{1}$ of the total space of $\mathrm{SL}_{n}(\mathbb{C}) \rightarrow \mathrm{SL}_{n}(\mathbb{C}) / P$ (cf. eq. (19)). In the quantum case we similarly have that $\mathcal{F}\left(U_{1}\right)^{\text {co } \mathcal{O}_{q}(P)} \subset \mathcal{F}\left(U_{1}\right)$ is a trivial HopfGalois extension. Recalling Remark 2.5 and Observation 2.7, we shall see it is the smashed product

$$
\mathcal{F}\left(U_{1}\right)=\mathbb{C}_{q}\left[a_{\alpha 1} a_{11}^{-1}\right]_{\alpha=2, \ldots n} \# \mathcal{O}_{q}(P),
$$

where the generators $\left(\begin{array}{cc}p_{11} & p_{1 \beta} \\ 0 & p_{\alpha \beta}\end{array}\right)$ of $\mathcal{O}_{q}(P)$ are identified with $\left(\begin{array}{cc}a_{11} & a_{1 \beta} \\ 0 & a_{11}^{-1} D_{1 \alpha}^{1 \beta}\end{array}\right)$.

The properties of $j_{1}: \mathcal{O}_{q}(P) \rightarrow \mathcal{F}\left(U_{1}\right)$ follow from the properties of an associated lift $J_{1}$ that maps into the localization $\mathcal{O}_{q}\left(\mathrm{M}_{n}\right)\left[a_{11}^{-1}\right]$ of the quantum matrix algebra defined in (15).

Lemma 5.4. Let $\mathcal{O}_{q}\left(p_{i j}\right)$ denote the quantum matrix algebra with generators $p_{i j}=$ $p_{11}, p_{1 \beta}, p_{\alpha \beta}$ and $p_{\alpha 1}=0 ; \alpha, \beta=2, \ldots, n$. We have a well defined algebra map $J_{1}: \mathcal{O}_{q}\left(p_{i j}\right) \longrightarrow \mathcal{O}_{q}\left(\mathrm{M}_{n}\right)\left[a_{11}^{-1}\right]$, that on the generators reads

$$
J_{1}\left(p_{11}^{ \pm 1}\right)=a_{11}^{ \pm 1}, \quad J_{1}\left(p_{1 \beta}\right)=a_{1 \beta}, \quad J_{1}\left(p_{\alpha \beta}\right)=a_{11}^{-1} D_{1 \alpha}^{1 \beta} .
$$


Proof. Recall, from [16], the following commutation relations in $\mathcal{O}_{q}\left(\mathrm{M}_{n}\right)$ among quantum determinants and generators of the algebra of quantum matrices:

$$
\begin{aligned}
& a_{1 \beta} D_{1 \alpha}^{1 \beta}=D_{1 \alpha}^{1 \beta} a_{1 \beta}, \quad a_{1 \gamma} D_{1 \alpha}^{1 \beta}=q D_{1 \alpha}^{1 \beta} a_{1 \gamma}, \quad \gamma>\beta \\
& a_{1 \gamma} D_{1 \alpha}^{1 \beta}=q D_{1 \alpha}^{1 \beta} a_{1 \gamma}+q\left(q^{-1}-q\right) D_{1 \beta}^{1 \gamma} a_{1 \alpha}, \quad \gamma<\beta
\end{aligned}
$$

where $D_{1 \alpha}^{1 \beta}=a_{11} a_{\alpha \beta}-q^{-1} a_{1 \beta} a_{\alpha 1}$. Also, by Theorem 7.3 in [16], the indeterminates $u_{\alpha \beta}:=D_{1 \alpha}^{1 \beta}$ satisfy the Manin relations as in Definition 5.1, where we replace $a_{\alpha \beta}$ with $u_{\alpha \beta}$. In order to show that $J_{1}$ is an algebra map, we have to show it is well defined. First, we easily compute the commutation relations

$$
a_{11}^{ \pm 1} D_{1 \alpha}^{1 \beta}=D_{1 \alpha}^{1 \beta} a_{11}^{ \pm 1}
$$

that imply that the $a_{11}^{-1} D_{1 \alpha}^{1 \beta}$, s satisfy the Manin relations among themselves. Next, we need to check that the commutation relations between $a_{1 \gamma}, \gamma=2, \ldots n$, and $a_{11}^{-1} D_{1 \alpha}^{1 \beta}$ are of the Manin kind.

If $\gamma>\beta$, we have:

$$
a_{1 \gamma} a_{11}^{-1} D_{1 \alpha}^{1 \beta}=a_{11}^{-1} D_{1 \alpha}^{1 \beta} a_{1 \gamma}
$$

because $a_{1 \gamma} a_{11}^{-1}=q^{-1} a_{11}^{-1} a_{1 \gamma}$ and $a_{1 \gamma} D_{1 \alpha}^{1 \beta}=q D_{1 \alpha}^{1 \beta} a_{1 \gamma}$.

If $\gamma=\beta$, we have:

$$
a_{1 \beta} a_{11}^{-1} D_{1 \alpha}^{1 \beta}=q^{-1} a_{11}^{-1} D_{1 \alpha}^{1 \beta} a_{1 \beta}
$$

because $a_{1 \beta}$ and $D_{1 \alpha}^{1 \beta}$ commute.

If $\gamma<\beta$, we need to check the commutation:

$$
a_{1 \gamma} a_{11}^{-1} D_{1 \alpha}^{1 \beta}=a_{11}^{-1} D_{1 \alpha}^{1 \beta} a_{1 \gamma}+\left(q^{-1}-q\right) a_{1 \beta} a_{11}^{-1} D_{1 \alpha}^{1 \gamma} .
$$

We leave this calculation as an exercise.

Lemma 5.5. Let the notation be as above. Let $\operatorname{det}_{q}\left(p_{i j}\right)$ and $\operatorname{det}_{q}\left(a_{i j}\right)$ denote respectively the quantum determinants in $\mathcal{O}_{q}\left(p_{i j}\right)$ and $\mathcal{O}_{q}\left(\mathrm{M}_{n}\right)\left[a_{11}^{-1}\right]$. Then

$$
J_{1}\left(\operatorname{det}_{q}\left(p_{i j}\right)\right)=\operatorname{det}_{q}\left(a_{i j}\right) .
$$

Proof. In the factorization (20), define:

$$
\left(b_{i j}\right):=\left(\begin{array}{cc}
1 & 0 \\
a_{\alpha 1} a_{11}^{-1} & 11
\end{array}\right) \quad\left(c_{i j}\right):=\left(\begin{array}{cc}
a_{11} & a_{1 \beta} \\
0 & a_{11}^{-1} D_{1 \alpha}^{1 \beta}
\end{array}\right)
$$

for $i, j=1, \ldots, n, \alpha, \beta=2, \ldots, n$. Since $c_{i j}=J_{1}\left(p_{i j}\right)$, by Lemma 5.4, they form a quantum matrix and our claim amounts to $\operatorname{det}_{q}\left(a_{i j}\right)=\operatorname{det}_{q}\left(c_{i j}\right)$.

We start by noticing that $b_{i j}$ and $c_{k l}$ satisfy the following commutation relations:

$$
\begin{gathered}
b_{i j} c_{k l}=c_{k l} b_{i j}, \quad j \neq 1, \quad b_{11} c_{k l}=c_{k l} b_{11}, \quad b_{i 1} c_{i l}=q^{-1} c_{i l} b_{i 1}, \quad i>1 \\
b_{i 1} c_{k l}=c_{k l} b_{i 1}, \quad k<i, \quad b_{i 1} c_{11}=q c_{11} b_{i 1} \\
b_{i 1} c_{k l}=c_{k l} b_{i 1}+\left(q^{-1}-q\right) c_{i l} b_{k 1}, \quad k>i .
\end{gathered}
$$


We also notice the obvious facts:

$$
b_{i i}=1, \quad b_{i j}=0, \quad i \neq j, \quad j \neq 1 .
$$

We proceed with a direct calculation of $\operatorname{det}_{q}\left(a_{i j}\right)$ using $a_{i j}=\sum_{k} b_{i k} c_{k j}$. Recall the quantum Laplace expansion along the first column (see [37] pg 47):

$$
\operatorname{det}_{q}\left(a_{i j}\right)=\sum_{r}(-q)^{-r+1} a_{r 1} A(r, 1)
$$

where $A(r, 1)$ is the quantum determinant obtained from $\left(a_{i j}\right)$ by removing the $r$-th row and first column,

$$
\begin{aligned}
\operatorname{det}_{q}\left(a_{i j}\right)= & a_{11} \sum_{\sigma}(-q)^{-\ell(\sigma)} a_{2 \sigma(2)} \ldots a_{n \sigma(n)} \\
& +\sum_{t=2}^{n}(-q)^{1-t} a_{t 1} \sum_{\sigma_{t}}(-q)^{-\ell\left(\sigma_{t}\right)} a_{1 \sigma_{t}(1)} \ldots \widehat{a_{t \sigma_{t}(t)}} \ldots a_{n \sigma(n)} \\
= & c_{11} \sum_{\sigma}(-q)^{-\ell(\sigma)} b_{2 k_{2}} c_{k_{2} \sigma(2)} \ldots b_{n k_{n}} c_{k_{n} \sigma(n)} \\
& +\sum_{t=2}^{n}(-q)^{1-t} b_{t 1} c_{11}^{-1} \sum_{\sigma_{t}, k_{1} \ldots \widehat{k}_{t}, \ldots k_{n}}(-q)^{-\ell\left(\sigma_{t}\right)} \\
& b_{1 k_{1}} c_{k_{1} \sigma(1)} \ldots b_{1 k_{t} c_{k_{t} \sigma(t)}} \ldots b_{n k_{n}} c_{k_{n} \sigma(n)},
\end{aligned}
$$

where $\widehat{u}$ means that we omit the term $u$.

Notice that $\sigma_{t}:\{1, \ldots, \widehat{t}, \ldots, n\} \longrightarrow\{2, \ldots, n\}$, but we treat it as a permutation, just renaming the elements of the two sets as the first $n-1$ natural numbers, so that $\ell\left(\sigma_{t}\right)$ is well defined.

Let us look at the term $b_{2 k_{2}} c_{k_{2} \sigma(2)} b_{3 k_{3}} c_{k_{3} \sigma(3)} \ldots b_{n k_{n}} c_{k_{n} \sigma(n)}$, where $k_{2}, \ldots, k_{n}=$ $1, \ldots, n$. We want to reorder it, and we claim that:

$$
b_{2 k_{2}} c_{k_{2} \sigma(2)} b_{3 k_{3}} c_{k_{3} \sigma(3)} \ldots b_{n k_{n}} c_{k_{n} \sigma(n)}=b_{2 k_{2}} b_{3 k_{3}} \ldots b_{n k_{n}} c_{k_{2} \sigma(2)} c_{k_{3} \sigma(3)} c_{k_{n} \sigma(n)} \text {. }
$$

By (23) $b_{2 k_{2}} \neq 0$ if and only if $k_{2}=1,2$. So we have to reorder $c_{k_{2} \sigma(2)} b_{3 k_{3}}$ only for $k_{2}<3$, hence, by (22), we have that they commute. The rest follows by repeated application of this argument.

Therefore, we can write the first term in (24) as:

$$
\begin{aligned}
a_{11} \sum_{\sigma}(-q)^{-\ell(\sigma)} a_{2 \sigma(2)} \ldots a_{n \sigma(n)} \\
\quad=c_{11} \sum_{\sigma, k_{2}, \ldots k_{n}}(-q)^{-\ell(\sigma)} b_{2 k_{2}} c_{k_{2} \sigma(2)} \ldots b_{n k_{n}} c_{k_{n} \sigma(n)} \\
\quad=c_{11} \sum_{\sigma, k_{2}, \ldots, k_{n}}(-q)^{-\ell(\sigma)} b_{2 k_{2}} b_{3 k_{3}} \ldots b_{n k_{n}} c_{k_{2} \sigma(2)} \ldots c_{k_{n} \sigma(n)} \\
\quad=c_{11} \sum_{k_{2}, \ldots, k_{n}} b_{2 k_{2}} b_{3 k_{3}} \ldots b_{n k_{n}} \sum_{\sigma}(-q)^{-\ell(\sigma)} c_{k_{2} \sigma(2)} \ldots c_{k_{n} \sigma(n)} \\
\quad=c_{11} \sum_{k_{2}, \ldots, k_{n}} b_{2 k_{2}} b_{3 k_{3}} \ldots b_{n k_{n}} C\left[k_{2}, \ldots, k_{n} \mid 2, \ldots, n\right]
\end{aligned}
$$


where $C\left[k_{2}, \ldots, k_{n} \mid 2, \ldots, n\right]$ is the quantum determinant in the indeterminates $c_{i j}$ obtained by taking rows $\left(k_{2}, \ldots, k_{n}\right)$ (in this order) and columns $(2, \ldots, n)$. Notice that, by (23), the sum over the index $k_{t}$ runs only on the values $k_{t}=1$ and $t$. If $k_{u}=k_{v}=1$ for some $u, v=2, \ldots, n$, then by Corollary 4.4 .4 in [37], we have $C\left[k_{2}, \ldots, k_{n} \mid 2, \ldots, n\right]=0$; so we must have $n-1$ distinct indices $1 \leq k_{2}, \ldots, k_{n} \leq n$ and $k_{u}=1$ for at most one of them.

We rewrite the first term in (24) as:

$$
\begin{aligned}
a_{11} \sum_{\sigma}( & -q)^{-\ell(\sigma)} a_{2 \sigma(2)} \ldots a_{n \sigma(n)} \\
= & c_{11} C[2, \ldots, n \mid 2, \ldots, n]+c_{11} b_{21} C[1,3, \ldots, n \mid 2, \ldots, n] \\
& \quad+c_{11} b_{31} C[2,1,4 \ldots, n \mid 2, \ldots, n]+c_{11} b_{41} C[2,3,1,5 \ldots, n \mid 2, \ldots, n] \\
& \quad+\ldots+c_{11} b_{n 1} C[2,3 \ldots, n-1,1 \mid 2, \ldots, n] \\
= & c_{11} C[2, \ldots, n \mid 2, \ldots, n]+\sum_{t}(-q)^{2-t} c_{11} b_{t 1} C[1, \ldots \widehat{t} \ldots, n \mid 2, \ldots, n] .
\end{aligned}
$$

Let us now look at the second term in (24). Reasoning as before, we have:

$$
\begin{aligned}
& (-q)^{1-t} a_{t 1} \sum_{\tau}(-q)^{-\ell(\tau)} a_{1 \tau(1)} \ldots \widehat{a_{t \tau(t)}} \ldots a_{n \tau(n)} \\
& =(-q)^{1-t} b_{t 1} c_{11} \sum_{\tau, k_{1}, \ldots k_{t} \ldots k_{n}}(-q)^{-\ell(\tau)} b_{1 k_{1}} c_{k_{1} \tau(1)} \ldots \widehat{b_{1 k_{t} c_{k_{t} \tau} \tau(t)} \ldots b_{n k_{n}} c_{n \tau(n)} .}
\end{aligned}
$$

However, we notice that here it must be $k_{1}=1$, otherwise $b_{1 k_{1}}=0$, hence this forces $k_{t}=t$ for all $t>1$. So we can write:

$$
\begin{aligned}
(-q)^{1-t} & a_{t 1} \sum_{\tau}(-q)^{-\ell(\tau)} a_{1 \tau(1)} \ldots \widehat{a_{t \tau(t)}} \ldots a_{n \tau(n)} \\
& =(-q)^{1-t} b_{t 1} c_{11} \sum_{\tau}(-q)^{-\ell(\tau)} c_{1 \tau(1)} \ldots \widehat{c_{t \tau(t)}} \ldots c_{n \tau(n)} \\
& =-(-q)^{2-t} c_{11} b_{t 1} C[1, \ldots \widehat{t}, \ldots, n \mid 2, \ldots, n]
\end{aligned}
$$

because by (22) we have $b_{t 1} c_{11}=q c_{11} b_{t 1}$.

If we substitute expressions (26) and (28) in (24) and simplify we remain with just one term:

$$
\operatorname{det}_{q}\left(a_{i j}\right)=c_{11} C[2, \ldots, n \mid 2, \ldots, n]=\operatorname{det}_{q}\left(c_{i j}\right) .
$$

Proposition 5.6. The map $j_{1}: \mathcal{O}_{q}(P) \longrightarrow \mathcal{F}\left(U_{1}\right):=\mathcal{O}_{q}\left(\mathrm{SL}_{n}\right)\left[a_{11}^{-1}\right]$ defined on the generators as:

$$
j_{1}\left(p_{11}^{ \pm 1}\right)=a_{11}^{ \pm 1}, \quad j_{1}\left(p_{1 \beta}\right)=a_{1 \beta}, \quad j_{1}\left(p_{\alpha \beta}\right)=a_{11}^{-1} D_{1 \alpha}^{1 \beta},
$$

$\alpha, \beta=2, \ldots n$, is an $\mathcal{O}_{q}(P)$-comodule algebra map. 
Proof. We canonically have $\mathcal{O}_{q}\left(\mathrm{SL}_{n}\right)\left[a_{11}^{-1}\right]=\mathcal{O}_{q}\left(\mathrm{M}_{n}\right)\left[a_{11}^{-1}\right] /\left(\operatorname{det}_{q}\left(a_{i j}\right)-1\right)$ and $\mathcal{O}_{q}(P)=$ $\mathcal{O}_{q}\left(\mathrm{SL}_{n}\right) / I_{q}(P)=\mathcal{O}_{q}\left(p_{i j}\right) /\left(\operatorname{det}_{q}\left(p_{i j}\right)-1\right)$ as algebras. Because of the previous lemma, $j_{1}: \mathcal{O}_{q}(P) \rightarrow \mathcal{O}_{q}\left(\mathrm{SL}_{n}\right)\left[a_{11}^{-1}\right]$ is well defined; in fact it is the algebra map $J_{1}: \mathcal{O}_{q}\left(p_{i j}\right) \rightarrow \mathcal{O}_{q}\left(\mathrm{M}_{n}\right)\left[a_{11}^{-1}\right]$ induced on the quotients.

We next show that $j_{1}$ is an $\mathcal{O}_{q}(P)$-comodule morphism, i.e., $\delta_{1} \circ j_{1}=\left(j_{1} \otimes\right.$ id $) \circ \Delta_{P}$, where $\Delta_{P}$ is the comultiplication in $\mathcal{O}_{q}(P)$ and $\delta_{1}$ is the $\mathcal{O}_{q}(P)$ coaction on $\mathcal{F}\left(U_{1}\right)=$ $\mathcal{O}\left(V_{1}\right)$ as defined in Proposition 4.3. Since $j_{1}$ is an algebra map, it is enough to check the comodule property on the generators. Let us look at the case of $p_{\alpha \beta}$, the case $p_{1 j}$ being an easy calculation. On the one hand, using the coproduct formula for quantum minors (see e.g. [16])

$$
\Delta\left(D_{1 i}^{1 j}\right)=\sum_{r<s} D_{1 i}^{r s} \otimes D_{r s}^{1 j}
$$

we have:

$$
\begin{aligned}
\left(\delta_{1} \circ j_{1}\right)\left(p_{\alpha \beta}\right) & =\delta_{1}\left(a_{11}^{-1}\right) \delta_{1}\left(D_{1 \alpha}^{1 \beta}\right)=\left(a_{11}^{-1} \otimes \pi\left(a_{11}^{-1}\right)\right) \sum_{r<s} D_{1 \alpha}^{r s} \otimes \pi\left(D_{r s}^{1 \beta}\right) \\
& =\sum_{k<\gamma} a_{11}^{-1} D_{1 \alpha}^{r \gamma} \otimes \pi\left(a_{11}^{-1} D_{r \gamma}^{1 \beta}\right)=\sum_{\gamma} a_{11}^{-1} D_{1 \alpha}^{1 \gamma} \otimes p_{\gamma \beta} .
\end{aligned}
$$

On the other hand:

$$
\left(\left(j_{1} \otimes \mathrm{id}\right) \circ \Delta_{P}\right)\left(p_{\alpha \beta}\right)=\left(j_{1} \otimes \mathrm{id}\right) \sum_{\gamma} p_{\alpha \gamma} \otimes p_{\gamma \beta}=\sum_{\gamma} a_{11}^{-1} D_{1 \alpha}^{1 \gamma} \otimes p_{\gamma \beta}
$$

We now extend the previous proposition in order to define the $\mathcal{O}_{q}(P)$-comodule algebra maps $j_{k}: \mathcal{O}_{q}(P) \rightarrow \mathcal{F}\left(U_{k}\right)=\mathcal{O}_{q}\left(\mathrm{SL}_{n}\right)\left[a_{k 1}^{-1}\right],(k=1, \ldots n)$ thus proving the triviality of the Hopf-Galois extensions $\mathcal{F}\left(U_{k}\right)^{\operatorname{co} \mathcal{O}_{q}(P)} \subset \mathcal{F}\left(U_{k}\right)$.

Reasoning as before, for each fixed value of $k$, we consider the factorization of quantum matrices $\left(a_{i j}\right)$ similar to (20):

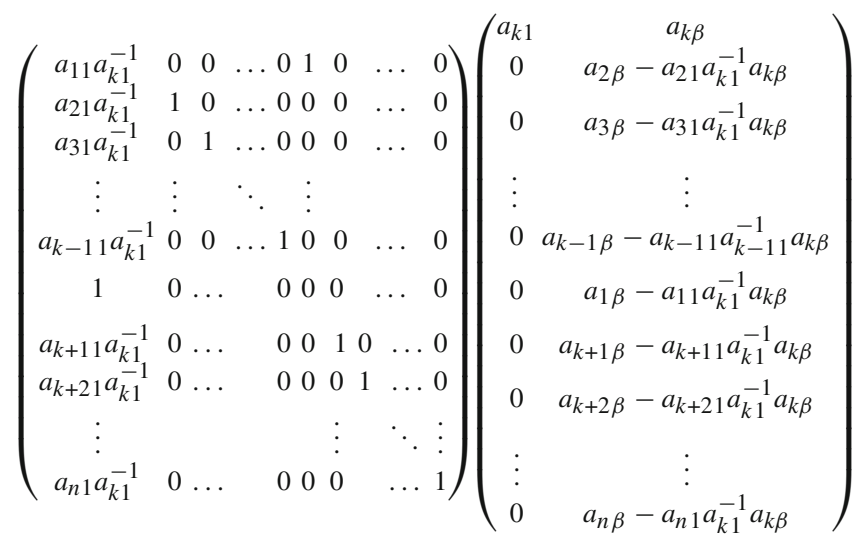

where $\beta=2, \ldots n$. This suggests to exchange row $k$ with row 1 in order to identify the last matrix with the matrix of generators $\left(\begin{array}{cc}p_{11} & p_{1 \beta} \\ 0 & p_{\alpha \beta}\end{array}\right)$ of $\mathcal{O}_{q}(P)$. 
Proposition 5.7. The map $j_{k}: \mathcal{O}_{q}(P) \longrightarrow \mathcal{F}\left(U_{k}\right)=\mathcal{O}_{q}\left(\mathrm{SL}_{n}\right)\left[a_{k 1}^{-1}\right]$, defined on the generators as:

$$
\begin{aligned}
& j_{k}\left(p_{11}^{ \pm 1}\right)=a_{k 1}^{ \pm 1}, \quad j_{k}\left(p_{1 \beta}\right)=a_{k \beta}, \quad j_{k}\left(p_{\alpha \beta}\right)=\left\{\begin{array}{c}
-q^{-1}\left(a_{1 \beta}-a_{11} a_{k 1}^{-1} a_{k \beta}\right) \alpha=k \\
a_{\alpha \beta}-a_{\alpha 1} a_{k 1}^{-1} a_{k \beta} \quad \alpha \neq k
\end{array},\right. \\
& \text { i.e., equivalently, } j_{k}\left(p_{\alpha \beta}\right)=\left\{\begin{array}{ll}
-q D_{\alpha k}^{1 \beta} a_{k 1}^{-1}, & \alpha<k \\
D_{1 k}^{1 \beta} a_{k 1}^{-1}, & \alpha=k \\
D_{k \alpha}^{1 \beta} a_{k 1}^{-1}, & \alpha>k
\end{array},\right.
\end{aligned}
$$

$\alpha, \beta=2, \ldots n$, and extended as algebra map to all $\mathcal{O}_{q}(P)$, is a well defined $\mathcal{O}_{q}(P)$ comodule algebra map for any $k=1, \ldots n$.

Proof. This is a direct check similar to Proposition 5.4. Recalling the commutation relations of the $p_{i j}$ 's (cf. proof of Proposition 5.4), and those between quantum minors in [16], we have: i) The $a_{k j}$ among themselves have the same commutation relations as the $p_{1 j}$ 's. ii) $a_{k 1}$ commutes with $D_{\alpha k}^{1 \beta}, D_{1 k}^{1 \beta}, D_{k \alpha}^{1 \beta}$. iii) The $-q a_{k 1}^{-1} D_{\alpha k}^{1 \beta}$, s, satisfy the same Manin relations among themselves as the $p_{\alpha \beta}$ 's; similarly for the $a_{k 1}^{-1} D_{1 k}^{1 \beta}$, s and the $a_{k 1}^{-1} D_{k \alpha}^{1 \beta}$, s. iv) The mixed commutation relations: of $-q a_{k 1}^{-1} D_{\alpha k}^{1 \beta}$ with $a_{k 1}^{-1} D_{1 k}^{1 \beta}$ and with $a_{k 1}^{-1} D_{k \alpha}^{1 \beta}$, and of $a_{k 1}^{-1} D_{1 k}^{1 \beta}$ with $a_{k 1}^{-1} D_{k \alpha}^{1 \beta}$, also satisfy the same Manin relations as those of the corresponding $p_{\alpha \beta}$ 's.

Then we are left to check the commutation relations of $a_{k \gamma}$ with $-q a_{k 1}^{-1} D_{\alpha k}^{1 \beta}, a_{k 1}^{-1} D_{1 k}^{1 \beta}$ and $a_{k 1}^{-1} D_{k \alpha}^{1 \beta}$. There are nine of these, depending on the combinations of $k>\alpha, k=$ $\alpha, k<\alpha$ with $\gamma>\beta, \gamma=\beta, \gamma<\beta$. These indeed correspond to the commutation relations between $p_{1 \gamma}$ and $p_{\alpha \beta}$.

We conclude that $j_{k}$ is a well defined algebra map because in $\mathcal{O}_{q}\left(\mathrm{SL}_{n}\right)\left[a_{k 1}^{-1}\right]$ we have $j_{k}\left(p_{11}\right) j_{k}\left(\operatorname{det}_{q}\left(p_{\alpha \beta}\right)\right)=1$, consistently with the last of the defining relations of the algebra $\mathcal{O}_{q}(P): p_{11} \operatorname{det}_{q}\left(p_{\alpha \beta}\right)=1$. This is obtained with the same argument as in Lemma 5.5.

Since $j_{k}$ is an algebra map it is an $\mathcal{O}_{q}(P)$-comodule map provided the comodule property $\delta_{1} \circ j_{1}=\left(j_{1} \otimes \mathrm{id}\right) \circ \Delta_{P}$ holds on the generators. It is straighforward to see that this is indeed the case on $p_{1 j}$. Let's compute the case $p_{\alpha \beta}$ with $\alpha>k$ (the other cases being similar):

$$
\begin{aligned}
\left(\delta_{k} \circ j_{k}\right)\left(p_{\alpha \beta}\right) & =\delta_{k}\left(a_{k 1}^{-1}\right) \delta_{k}\left(D_{k \alpha}^{1 \beta}\right)=\left(a_{k 1}^{-1} \otimes \pi\left(a_{11}^{-1}\right)\right) \sum_{r<s} D_{k \alpha}^{r s} \otimes \pi\left(D_{r s}^{1 \beta}\right) \\
& =\sum_{r<\gamma} a_{k 1}^{-1} D_{1 \alpha}^{r \gamma} \otimes \pi\left(a_{11}^{-1} D_{r \gamma}^{1 \beta}\right)=\sum_{\gamma} a_{k 1}^{-1} D_{1 \alpha}^{1 \gamma} \otimes p_{\gamma \beta} \\
& =\left(\left(j_{1} \otimes \mathrm{id}\right) \circ \Delta_{P}\right)\left(p_{\alpha \beta}\right) .
\end{aligned}
$$

Remark 5.8. Recalling Remark 2.5 and Observation 2.7, as corollary of the above proposition we have $\mathcal{F}\left(U_{k}\right) \simeq \mathbb{C}_{q}\left[a_{i 1} a_{k 1}^{-1}\right]_{i \in \mathcal{I}_{k}} \# \mathcal{O}_{q}(P), \mathcal{I}_{k}:=\{i \mid 1 \leq i \leq n, i \neq k\}$, where it is easy to check that the smashed product is nontrivial (i.e., different from the tensor product).

Theorem 5.9. Let the notation be as in the previous section, with $\mathcal{I}=\{1, \ldots n\}$. The assignment:

$$
U_{I} \mapsto \mathcal{F}\left(U_{I}\right):=\mathcal{O}_{q}\left(\mathrm{SL}_{n}\right) S_{i_{1}}^{-1} \ldots S_{i_{s}}^{-1}, \quad I=\left\{i_{1}, \ldots, i_{s}\right\}
$$


with $i_{1}, \ldots i_{s}, s \in \mathcal{I}$, defines a locally cleft $\mathcal{O}_{q}(P)$-principal bundle on the quantum ringed space $\left(\mathrm{SL}_{n}(\mathbb{C}) / P, \mathcal{F}^{\mathrm{co} \mathcal{O}_{q}(P)}\right)$, with structure sheaf $\mathcal{F}^{\mathrm{co} \mathcal{O}_{q}(P)}=\mathcal{O}_{\mathrm{SL}_{n} / P}$ given by projective localizations of the quantum homogeneous projective space $\tilde{\mathcal{O}}_{q}\left(\mathbf{P}^{n-1}\right)=$ $\tilde{\mathcal{O}}_{q}\left(\mathrm{SL}_{n} / P\right)$.

Proof. After Proposition 5.3 we only need to prove the locally cleft property. This is a direct consequence of Proposition 5.7 and Remark 2.5.

Remark 5.10. Notice that our construction, and in particular Theorem 5.9, holds also when we specialize the indeterminate $q$ to a complex value $q \in \mathbb{C}$ that is not a root of unity. Then locally cleft sheaves are locally principal (cf. Definition 2.9 and Definition 2.10) and hence the sheaf $U_{I} \mapsto \mathcal{F}\left(U_{I}\right)$ of Theorem 5.9 is a quantum principal bundle on quantum projective space.

\section{Quantum Principal Bundles from Twists}

In this section we obtain new quantum principal bundles via 2-cocycle (twist) deformations. In particular we provide examples that are locally cleft from examples that are locally trivial.

We here consider the ground ring to be a field, hence specialize $q \in k$. As in [2] we consider 2-cocycle deformations based on the "structure group" Hopf algebra $H$ and also on an "external symmetry" Hopf algebra $K$, i.e. a Hopf algebra coacting on the quantum principal bundle, the coaction being compatible with that of $H$ (in the commutative case $K$ is associated with automorphisms of the bundle, possibly nontrivial on the base).

This general theory of twists of quantum principal bundles leads in particular to principal bundles on multiparametric quantum projective spaces. It can also be adapted to recover the $\theta$-twisted multipullback $n-1$-dimensional projective space studied in [24], indeed this latter is obtained as the fixed point subalgebra under a free $U(1)$-action of a "non round" $2 n-1$-dimensional $\theta$-twisted sphere. It is obtained via multipullbacks based on a closed covering which is a restriction of our usual open affine covering of $\mathbf{P}^{n-1}(\mathbb{C})$.

6.1. Deformations from twists of $H$. Let $\gamma: H \otimes H \rightarrow k$ be a 2-cocycle of the Hopf algebra $H$, denote by $\gamma^{-1}: H \otimes H \rightarrow k$ its convolution inverse and by $H_{\gamma}$ the new Hopf algebra that has the same costructures of $H$ and new product $\cdot \gamma$ and antipode obtained by twisting the ones of $H$ via $\gamma$. Explicitly, the product reads, for all $h, h^{\prime} \in H$, $h \cdot{ }_{\gamma} h^{\prime}=\gamma\left(h_{(1)} \otimes h_{(1)}^{\prime}\right) h_{(2)} h_{(2)}^{\prime} \gamma^{-1}\left(h_{(3)} \otimes h_{(3)}^{\prime}\right)$. We also denote with $\Gamma$ the functor from the category of right $H$-comodule algebras to that of right $H_{\gamma}$-comodule algebras: if $A$ is an $H$-comodule algebra then $\Gamma(A) \equiv A_{\gamma}$ is the $k_{q}$-module $A$ with product $a \bullet \gamma a^{\prime}:=a_{(0)} a_{(0)}^{\prime} \gamma^{-1}\left(a_{(1)} \otimes a_{(1)}^{\prime}\right)$. Since $H$ and $H_{\gamma}$ have the same costructures, $A_{\gamma}$ is a right $H_{\gamma}$-comodule algebra using the same comodule structure map as for $A$. The functor $\Gamma$ is the identity on morphisms.

Furthermore, the convolution inverse $\gamma^{-1}$ is a 2-cocycle of $H_{\gamma}$; we have $\left(H_{\gamma}\right)_{\gamma^{-1}}=$ $H$ and similarly we can twist back the $H_{\gamma}$-comodule algebra $A_{\gamma}$ to the $H_{\gamma}$-comodule algebra $\Gamma^{-1}\left(A_{\gamma}\right) \equiv\left(A_{\gamma}\right)_{\gamma^{-1}}=A$. This implies that the functor $\Gamma$ and its inverse $\Gamma^{-1}$ are an equivalence (actually an isomorphism) between the categories of $H$-comodule algebras and $H_{\gamma}$-comodule algebras. 
Theorem 6.1. Let $\gamma$ be a 2-cocycle of the Hopf algebra $H$ and $\Gamma$ the corresponding functor of comodule algebras. The sheaf $\mathcal{F}$ is an $H$-principal bundle (quantum principal bundle) over the ringed space $\left(M, \mathcal{O}_{M}\right)$ if and only if $\Gamma \circ \mathcal{F}$ is an $H_{\gamma}$-principal bundle over $\left(M, \mathcal{O}_{M}\right)$. The sheaf $\mathcal{F}$ is a locally cleft quantum principal bundle if and only if so is $\Gamma \circ \mathcal{F}$.

Proof. If $\mathcal{F}$ is a sheaf of $H$-comodule algebras over $M$ then $\Gamma \circ \mathcal{F}$ is a sheaf of $H_{\gamma^{-}}$ comodule algebras over $M$, indeed, presheaf, locality and the gluing properties immediately follow recalling that the functor $\Gamma$ has inverse functor $\Gamma^{-1}$. For any open $U \subset M$, since $H_{\gamma}$ and $H$ have the same coproduct, we have $\mathcal{F}(U)_{\gamma}^{\operatorname{co} H_{\gamma}}=\mathcal{F}(U)^{\operatorname{co} H}=\mathcal{O}_{M}(U)$ as comodules and hence as algebras (multiplication of coinvariant elements is undeformed). This shows that $\Gamma \circ \mathcal{F}$ is a sheaf over the ringed space $\left(M, \mathcal{O}_{M}\right)$. Vice versa, if $\Gamma \circ \mathcal{F}$ is a sheaf of $H_{\gamma}$-comodule algebras over the ringed space $\left(M, \mathcal{O}_{M}\right)$ then $\mathcal{F}=\Gamma^{-1} \circ(\Gamma \circ \mathcal{F})$ is a sheaf of $H$-comodule algebras over $\left(M, \mathcal{O}_{M}\right)$.

Finally, let $\left\{U_{i}\right\}$ be a covering of $M$ such that $\mathcal{F}$ is locally principal (locally cleft) over $\left\{U_{i}\right\}$. The extension $\mathcal{O}_{M}\left(U_{i}\right)=\mathcal{F}\left(U_{i}\right)^{\mathrm{co} H} \subset \mathcal{F}\left(U_{i}\right)$ is principal (cleft) if and only if $\mathcal{O}_{M}\left(U_{i}\right)=\mathcal{F}\left(U_{i}\right)_{\gamma}^{\mathrm{co} H_{\gamma}} \subset \mathcal{F}\left(U_{i}\right)_{\gamma}$ is principal (cleft), cf. [2, Corollary 3.9] (cf. [32, Theorem 5.2] or [2, Corollary 3.7]).

Remark 6.2. We further observe that if the $H$-principal bundle $\mathcal{F}$ is locally trivial with respect to a covering $\left\{U_{i}\right\}$, i.e., the cleft extensions $\mathcal{F}\left(U_{i}\right)^{\operatorname{co} H} \subset \mathcal{F}\left(U_{i}\right)$ are trivial extensions, so that $\mathcal{F}\left(U_{i}\right) \simeq \mathcal{F}\left(U_{i}\right)^{\operatorname{co} H} \sharp H$ (cf. Observation 2.7), then this is no more the case for the twisted $H_{\gamma}$-principal bundle $\Gamma \circ \mathcal{F}$ because the extensions $\mathcal{F}\left(U_{i}\right)^{\operatorname{co} H_{\gamma}} \subset$ $\mathcal{F}\left(U_{i}\right)_{\gamma}$ are cleft but nontrivial. Indeed, as follows from [32, Theorem 5.2], $\mathcal{F}\left(U_{i}\right)_{\gamma} \simeq$ $\mathcal{F}\left(U_{i}\right)^{\mathrm{co} H_{\gamma^{-1}}} H_{\gamma}$, where $\sharp_{\gamma^{-1}}$ denotes the crossed product given by the 2-cocyle $\gamma^{-1}$ of $H_{\gamma}$.

6.2. Deformations from twists of $K$. Let now $K$ be another Hopf algebra and $\mathcal{F}$ be a sheaf over the ringed space $\left(M, \mathcal{O}_{M}\right)$ of $(K, H)$-bicomodule algebras, i.e. right $H$-comodule algebras and left $K$-comodule algebras with left and right coactions commuting: $(\rho \otimes$ $i d) \circ \delta=(i d \otimes \delta) \circ \rho$. Since $k$ is a field, $K$ is free as a $k$-module and $\mathcal{F}^{\mathrm{co} H}: U \rightarrow \mathcal{F}(U)^{\mathrm{co} H}$ is a subsheaf of $K$-comodule algebras (because $\mathcal{F}(U)^{\mathrm{co} H}$ are $K$-subcomodule algebras, cf. [2, Proposition 3.12]).

A twist $\sigma$ of $K$ gives the functor $\Sigma$ from left $K$-comodule algebras $A$ to left $K_{\sigma^{-}}$ comodule algebras $\Sigma(A) \equiv{ }_{\sigma} A$, where the new product is given by $a_{\sigma} \bullet a^{\prime}=\sigma\left(a_{(-1)} \otimes\right.$ $\left.a_{(-1)}^{\prime}\right) a_{(0)} a_{(0)}^{\prime}$ (the comodule structure maps of $A$ and $\sigma A$ being the same). The functor $\Sigma$ is the identity on morphisms. The convolution inverse $\sigma^{-1}$ is a twist of ${ }_{\sigma} K$ and gives the inverse functor $\Sigma^{-1}$. As in Theorem 6.1, composition of the functor $\Sigma$ with the sheaf $\mathcal{F}$ of $(K, H)$-bicomodule algebras gives the sheaf $\Sigma \circ \mathcal{F}$ of $\left(K_{\sigma}, H\right)$-bicomodule algebras.

Theorem 6.3. Let the sheaf $\mathcal{F}$ of $(K, H)$-bicomodule algebras over the ringed space $\left(M, \mathcal{F}^{\mathrm{co} H}\right)$ be an $H$-principal bundle. Then the sheaf $\Sigma \circ \mathcal{F}$ of $\left(K_{\sigma}, H\right)$-bicomodule algebras over the ringed space $\left(M, \Sigma \circ \mathcal{F}^{\mathrm{co} H}\right)$ is an $H$-principal bundle.

Furthermore, if $\mathcal{F}$ is a locally cleft quantum principal bundle (relative to a covering $\left\{U_{i}\right\}$ of $\left.M\right)$ and if the $H$-comodule $(H, \Delta)$ has a compatible $K$-comodule structure, so that it is a $(K, H)$-bicomodule and the cleaving maps $j_{i}: H \rightarrow \mathcal{F}\left(U_{i}\right)$ are $(K, H)$ bicomodule maps, then the sheaf $\Sigma \circ \mathcal{F}$ is a locally cleft quantum principal bundle. 
Proof. As in Theorem 6.1 invertibility of $\Sigma$ implies that $\Sigma \circ \mathcal{F}$ and $\Sigma \circ \mathcal{F}^{\mathrm{co} H}$ are sheaves of $\left(K_{\sigma}, H\right)$-comodule algebras and of $K_{\sigma}$-comodule algebras, respectively. For all $U \subset M, \Sigma \circ \mathcal{F}^{\mathrm{co} H}(U)=(\Sigma \circ \mathcal{F})^{\mathrm{co} H}(U)$ as $K_{\sigma}$-comodules (indeed $\Sigma$ twists only the algebra structure), they are also equal as $K_{\sigma}$-comodule algebras because $\mathcal{F}^{\text {co } H}(U)$ is a $K$-subcomodule of $\mathcal{F}(U)$. This shows the equality $\Sigma \circ \mathcal{F}^{\operatorname{co} H}=(\Sigma \circ \mathcal{F})^{\operatorname{co} H}$ as sheaves of $K_{\sigma}$-comodule algebras.

Since the 2-cocycle $\sigma$ twists $H$-principal $(H, K)$-comodule algebras $\mathcal{F}(U)$ in $H$ principal $\left(H, K_{\sigma}\right)$-comodule algebras $\sigma_{\sigma} \mathcal{F}(U)$, see [2, Corollary 3.19], we have that the sheaf $\Sigma \circ \mathcal{F}$ is locally $H$-principal, hence it is a quantum principal bundle over the ringed space $\left(M, \Sigma \circ \mathcal{F}^{\mathrm{coH}}\right)$.

We are left to show that in the further hypotheses regarding the locally cleft property of $\mathcal{F}$ we have that $\Sigma \circ \mathcal{F}$ is locally cleft. From Theorem 2.6, for each open $U_{i}$ we have the local trivialization

$$
\vartheta_{i}: \mathcal{F}\left(U_{i}\right)^{\mathrm{co} H} \otimes H \rightarrow \mathcal{F}\left(U_{i}\right), \quad b \otimes h \mapsto \vartheta_{i}(b \otimes h)=b j_{i}(h)
$$

that is an isomorphism of left $\mathcal{F}\left(U_{i}\right)^{\mathrm{co} H}$-modules and right $H$-comodules. Since $j_{i}$ is also a left $K$-module map and $\mathcal{F}\left(U_{i}\right)$ is a $K$-comodule algebra we easily have that $\vartheta_{i}$ is also a left $K$-comodule map.

Recall that a twist $\sigma$ defines a monoidal functor $\left(\Sigma, \varphi^{\ell}\right)$ from the category of left $K$-comodules $\left({ }^{K} \mathcal{M}, \otimes\right)$ to that of left $K_{\sigma}$-comodules $\left(K_{\sigma} \mathcal{M},{ }^{\sigma} \otimes\right)$, where ${ }^{\sigma} \otimes$ and $\otimes$ coincide as tensor products of $k$-modules. The functor $\Sigma:{ }^{K} \mathcal{M} \rightarrow{ }^{K}{ }_{\sigma} \mathcal{M}, V \mapsto$ $\Sigma(V) \equiv{ }_{\sigma} V$ is the identity on objects and morphisms, because as coalgebras $K=K_{\sigma}$, while the natural transformation $\varphi^{\ell}$ between the tensor product functors $\otimes$ and ${ }^{\sigma} \otimes$ is given by the ${ }_{\sigma} K$-comodule isomorphisms $\varphi_{V W}^{\ell}: \Sigma(V \otimes W) \rightarrow \Sigma(V)^{\sigma} \otimes \Sigma(W)$, $v \otimes w \mapsto \varphi_{M N}^{\ell}(v \otimes w)=\sigma\left(v_{(-1)} \otimes w_{(-1)}\right) v_{(0)} \otimes w_{(0)}$, where $\rho(v)=v_{(-1)} \otimes v_{(0)}$, $\rho(w)=w_{(-1)} \otimes w_{(0)}$ are the left $K$-coactions of $V$ and $W$.

Furthermore, $\left(\Sigma, \varphi^{\ell}\right)$ is a monoidal functor from the category of $(K, H)$-bicomodules $\left({ }^{K} \mathcal{M}^{H}, \otimes\right)$ to that of $\left(K_{\sigma}, H\right)$-bicomodules $\left(K_{\sigma} \mathcal{M}^{H},{ }^{\sigma} \otimes\right)$, (cf. for example [2, §2.2]).

Applying the functor $\Sigma$ to the $\mathcal{F}\left(U_{i}\right)^{\mathrm{co} H}$-module and $(K, H)$-bicomodule isomorphism $\vartheta_{i}$ we obtain the isomorphism of left ${ }_{\sigma} \mathcal{F}\left(U_{i}\right)^{\operatorname{co} H}$-modules and $\left(K_{\sigma}, H\right)$ bicomodules

$$
\Sigma\left(\vartheta_{i}\right):{ }_{\sigma}\left(\mathcal{F}\left(U_{i}\right)^{\mathrm{co} H} \otimes H\right) \rightarrow{ }_{\sigma} \mathcal{F}\left(U_{i}\right)
$$

where ${ }_{\sigma}\left(\mathcal{F}\left(U_{i}\right)^{\operatorname{co} H} \otimes H\right):=\Sigma\left(\mathcal{F}\left(U_{i}\right)^{\operatorname{co} H} \otimes H\right)$ and ${ }_{\sigma} \mathcal{F}\left(U_{i}\right):=\Sigma\left(\mathcal{F}\left(U_{i}\right)\right)$. Using the $\left(K_{\sigma}, H\right)$-bicomodule isomorphism (we suppress the lower indices of $\varphi^{\ell}$ for simplicity)

$$
\varphi^{\ell}:{ }_{\sigma}\left(\mathcal{F}\left(U_{i}\right)^{\operatorname{co} H} \otimes H\right) \rightarrow{ }_{\sigma} \mathcal{F}\left(U_{i}\right)^{\operatorname{co} H} \sigma_{\otimes} H
$$

where $_{\sigma} H:=\Sigma(H)$ is just the $(K, H)$-bicomodule $H$ now seen as a $\left(K_{\sigma}, H\right)$-bicomodule, we obtain the left ${ }_{\sigma} \mathcal{F}\left(U_{i}\right)^{\mathrm{co} H}$-module and $\left(K_{\sigma}, H\right)$-bicomodule isomorphism

$$
\Sigma\left(\vartheta_{i}\right) \circ \varphi^{\ell-1}:{ }_{\sigma} \mathcal{F}\left(U_{i}\right)^{\mathrm{co} H} \sigma_{\otimes} H \rightarrow{ }_{\sigma} \mathcal{F}\left(U_{i}\right)
$$

Forgetting the $K_{\sigma}$-comodule structure and recalling that as $H$-comodules ${ }_{\sigma} H=H$, and that as tensor products of $H$-comodules we have ${ }^{\sigma} \otimes=\otimes$, this isomorphism becomes an ${ }_{\sigma} \mathcal{F}\left(U_{i}\right)^{\operatorname{co} H}$-module and $H$-comodule isomorphism ${ }_{\sigma} \mathcal{F}\left(U_{i}\right)^{\operatorname{co} H} \otimes H \rightarrow{ }_{\sigma} \mathcal{F}\left(U_{i}\right)$, proving that the extension ${ }_{\sigma} \mathcal{F}\left(U_{i}\right)^{\operatorname{co} H} \subset{ }_{\sigma} \mathcal{F}\left(U_{i}\right)$ is cleft. This holds for each open $U_{i}$, thus $\Sigma \circ \mathcal{F}$ is locally cleft. 
6.3. Examples. We twist the quantum principal bundle $\mathcal{F}$ on the quantum ringed space

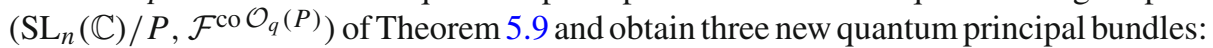
$\Gamma \circ \mathcal{F}, \Sigma \circ \mathcal{F}$ and $\Sigma \circ \Gamma \circ \mathcal{F}$; the first on the locally ringed space associated with the homogeneous ring of quantum projective space $\tilde{\mathcal{O}}_{q}\left(\mathbf{P}^{n-1}\right)$, the other two on its multiparametric deformation $\tilde{\mathcal{O}}_{q, \gamma}\left(\mathbf{P}^{n-1}\right)$.

Deformations from twists of $H=\mathcal{O}_{q}(P)$.

The $(n-1)$-dimensional torus $\mathbb{T}^{n-1}$ is a subgroup of $S L_{n}(\mathbb{C})$ and correspondingly we have that the Hopf algebra $\mathcal{O}\left(\mathbb{T}^{n-1}\right)$ (the group Hopf algebra over $\mathbb{C}$ of the free abelian group generated by $n-1$ elements) is a quotient of $\mathcal{O}_{q}\left(S L_{n}\right)$. It is useful to present $\mathcal{O}\left(\mathbb{T}^{n-1}\right)$ as the algebra over $\mathbb{C}$ generated by the $n$ elements $t_{i}, i=1, \ldots n$ and their inverses $t_{i}^{-1}$ modulo the ideal generated by the relation $t_{1} t_{2} \ldots t_{n}=1$. The Hopf algebra structure is fixed by requiring $t_{i}$ to be group like. The Hopf algebra projection $\mathcal{O}_{q}\left(S L_{n}\right) \stackrel{p r}{\longrightarrow} \mathcal{O}\left(\mathbb{T}^{n-1}\right)$ on the generators is given by

$$
a_{i j} \mapsto \delta_{i j} t_{i}
$$

We consider the exponential 2-cocycle $\gamma$ on $\mathcal{O}\left(\mathbb{T}^{n-1}\right)$ defined on the generators $t_{i}$ by

$$
\gamma\left(t_{j} \otimes t_{k}\right)=\gamma_{j k} \quad \text { with } \gamma_{j k}=\exp \left(i \pi \theta_{j k}\right) ; \quad \theta_{j k}=-\theta_{k j} \in \mathbb{R}
$$

and extended to the whole algebra via

$$
\gamma(a b \otimes c)=\gamma\left(a \otimes c_{(1)}\right) \gamma\left(b \otimes c_{(2)}\right), \gamma(a \otimes b c)=\gamma\left(a_{(1)} \otimes c\right) \gamma\left(a_{(2)} \otimes b\right)(32)
$$

for all $a, b, c, \in \mathcal{O}\left(\mathbb{T}^{n}\right)$. This 2-cocycle $\gamma$ is pulled back along the projection $\mathcal{O}_{q}\left(S L_{n}\right) \stackrel{p r}{\longrightarrow}$ $\mathcal{O}\left(\mathbb{T}^{n-1}\right)$ to a 2-cocycle $\gamma \circ(p r \otimes p r)$ on $\mathcal{O}\left(\mathrm{SL}_{n}\right)$ (see e.g. [2, Lemma 4.1]). Explicitly, denoting with abuse of notation by $\gamma$ the pulled back 2-cocycle, we have that

$$
\gamma: \mathcal{O}_{q}\left(S L_{n}\right) \otimes \mathcal{O}_{q}\left(S L_{n}\right) \rightarrow \mathbb{C}
$$

is defined by $\gamma\left(a_{i j} \otimes a_{k l}\right)=\delta_{i j} \delta_{k l} \gamma_{i l}$, and (32) for all $a, b, c \in \mathcal{O}_{q}\left(S L_{n}\right)$. Twist deformation via this 2-cocycle of the quantum group $\mathcal{O}_{q}\left(S L_{n}\right)$ gives the multiparametric special linear quantum group studied e.g. in [35].

The torus Hopf algebra $\mathcal{O}\left(\mathbb{T}^{n-1}\right)$ is also a quotient of the parabolic quantum group $\mathcal{O}_{q}(P)$ defined in (18). Correspondingly the 2-cocycle $\gamma$ on $\mathcal{O}\left(\mathbb{T}^{n-1}\right)$ is pulled back to a 2-cocycle, still denoted $\gamma$, on $\mathcal{O}_{q}(P)$ providing its multiparametric deformation $\mathcal{O}_{q, \gamma}(P)$.

We now apply Theorem 6.1 to the locally cleft $\mathcal{O}_{q}(P)$-principal bundle $\mathcal{F}$ on the quantum ringed space $\left(\mathrm{SL}_{n}(\mathbb{C}) / P, \mathcal{F}^{\text {co } \mathcal{O}_{q}(P)}\right)$ of Theorem 5.9 and obtain the $\mathcal{O}_{q, \gamma}(P)$ principal bundle $\Gamma \circ \mathcal{F}$ on $\left(\mathrm{SL}_{n}(\mathbb{C}) / P, \mathcal{F}^{\mathrm{co}} \mathcal{O}_{q}(P)\right)$. Furthermore, Remark 6.2 implies that while the $\mathcal{O}_{q}(P)$-principal bundle $\mathcal{F}$ is locally trivial on the cover $\left\{U_{i}\right\}$ of $\mathbf{P}^{n-1}(\mathbb{C})=$ $\mathrm{SL}_{n}(\mathbb{C}) / P$, the $\mathcal{O}_{q, \gamma}(P)$-principal bundle $\Gamma \circ \mathcal{F}$ is only locally cleft.

Deformations from twists of $K=\mathcal{O}\left(\mathbb{T}^{n-1}\right)$.

We next study twists based on the external Hopf algebra $K=\mathcal{O}\left(\mathbb{T}^{n-1}\right)$. The $\mathcal{O}_{q}(P)$ principal bundle $\mathcal{F}$ on $\left(\mathrm{SL}_{n}(\mathbb{C}) / P, \mathcal{F}^{\mathrm{co}} \mathcal{O}_{q}(P)\right)$ of Theorem 5.9 is indeed a sheaf of $\left(\mathcal{O}\left(\mathbb{T}^{n-1}\right), \mathcal{O}_{q}(P)\right)$-bicomodule algebras: The left $K=\mathcal{O}\left(\mathbb{T}^{n-1}\right)$-coaction on the $\mathcal{O}_{q}(P)$ comodule algebra $\mathcal{O}_{q}\left(\mathrm{SL}_{n}(\mathbb{C})\right)$ is given by

$$
\rho(a)=(p r \otimes i d) \Delta_{\mathcal{O}_{q}\left(\mathrm{SL}_{n}(\mathbb{C})\right)}(a)
$$


for all $a \in \mathcal{O}_{q}\left(\mathrm{SL}_{n}(\mathbb{C})\right)$, and is uniquely extended as algebra map to the sheaf $U_{I} \mapsto$ $\mathcal{F}\left(U_{I}\right)=\mathcal{O}_{q}\left(\mathrm{SL}_{n}(\mathbb{C})\right) S_{i_{1}}^{-1} \ldots S_{i_{s}}^{-1}, I=\left\{i_{1}, \ldots i_{s}\right\}$ of $\mathcal{O}_{q}(P)$-comodule algebras on $\mathrm{SL}_{n}(\mathbb{C}) / P$, where $\left\{U_{I}\right\}$, with $I \in\left\{\left\{i_{1}, \ldots i_{s}\right\} \mid i_{1}, \ldots i_{s}, s=1, \ldots n\right\}$, is the topology on $\mathrm{SL}_{n}(\mathbb{C}) / P$ generated by the cover $\left\{U_{i}\right\}, i=1, \ldots n$.

Furthermore, the cleaving maps $j_{i}: \mathcal{O}_{q}(P) \rightarrow \mathcal{F}\left(U_{i}\right)=\mathcal{O}_{q}\left(\mathrm{SL}_{n}(\mathbb{C})\right) S_{i}^{-1}$ become $\left(\mathcal{O}\left(\mathbb{T}^{n-1}\right), \mathcal{O}_{q}(P)\right)$-comodule maps by defining on the $\mathcal{O}_{q}(P)$-comodule $\left(\mathcal{O}_{q}(P), \Delta\right)$ the compatible left $\mathcal{O}\left(\mathbb{T}^{n-1}\right)$-comodule structure given by $\rho(a)=(p \otimes i d) \Delta(a)$, where $p$ is the projection $\mathcal{O}_{q}(P) \stackrel{p}{\longrightarrow} \mathcal{O}\left(\mathbb{T}^{n-1}\right)$. We can then consider the 2-cocycle (31) for $K=\mathcal{O}\left(\mathbb{T}^{n-1}\right)$ and apply Theorem 6.3 thus concluding that the sheaf $\Sigma \circ \mathcal{F}$ is a locally cleft $\mathcal{O}_{q}(P)$-principal bundle over the ringed space $\left(\mathbf{P}^{n-1}(\mathbb{C}), \Sigma \circ \mathcal{F}^{\operatorname{co} \mathcal{O}_{q}(P)}\right)$. In Remark 6.6 we further show it is not locally trivial on the cover $\left\{U_{i}\right\}$.

Deformations from both twists of $H=\mathcal{O}_{q}(P)$ and $K=\mathcal{O}\left(\mathbb{T}^{n-1}\right)$.

Finally, we can consider the $\mathcal{O}_{q}(P)$-principal bundle $\Sigma \circ \mathcal{F}$ over the ringed space $(M, \Sigma \circ$ $\left.\mathcal{F}^{\text {co } \mathcal{O}_{q}(P)}\right)$, and use the 2-cocycle of $\mathcal{O}_{q}(P)$, obtained via pullback of the 2-cocycle (31) of $\mathcal{O}\left(\mathbb{T}^{n-1}\right)$, in order to construct, according to Theorem 6.1, the $\mathcal{O}_{q, \gamma}(P)$-principal bundle $\Gamma \circ \Sigma \circ \mathcal{F}$ over the ringed space $\left(\mathbf{P}^{n-1}(\mathbb{C}), \Sigma \circ \mathcal{F}^{\mathrm{co} \mathcal{O}_{q}(P)}\right)$.

Equivalently, the quantum principal bundle $\Gamma \circ \Sigma \circ \mathcal{F}$ is over $\left(\mathbf{P}^{n-1}(\mathbb{C}), \Sigma \circ(\Gamma \circ\right.$ $\left.\mathcal{F})^{\operatorname{co}_{q, \gamma}(P)}\right)$, since $\left(\mathbf{P}^{n-1}(\mathbb{C}), \Sigma \circ \mathcal{F}^{\operatorname{co} \mathcal{O}_{q}(P)}\right)=\left(\mathbf{P}^{n-1}(\mathbb{C}), \Sigma \circ(\Gamma \circ \mathcal{F})^{\operatorname{co} \mathcal{O}_{q, \gamma}(P)}\right)$, as follows from $\mathcal{O}_{q, \gamma}(P)$ and $\mathcal{O}_{q}(P)$ having the same coproduct. In fact, since left and right coactions commute, we have $\Sigma \circ \Gamma=\Gamma \circ \Sigma$ (cf. [2, Proposition 2.27]) and therefore the equality

$$
\Sigma \circ \Gamma \circ \mathcal{F}=\Gamma \circ \Sigma \circ \mathcal{F}
$$

of quantum principal bundles over $\left(\mathbf{P}^{n-1}(\mathbb{C}), \Sigma \circ(\Gamma \circ \mathcal{F})^{\operatorname{co} \mathcal{O}_{q, \gamma}(P)}\right)$. We now study this $\mathcal{O}_{q, \gamma}(P)$-principal bundle, that is locally cleft since so is $\mathcal{F}$, and conclude by proving that since $\mathcal{F}$ is locally trivial so is $\Sigma \circ \Gamma \circ \mathcal{F}$.

We show that the $\mathcal{O}_{q, \gamma}(P)$-principal bundle $\Sigma \circ \Gamma \circ \mathcal{F}$ is an example of the construction of Theorem 4.8. This is so because the (graded) algebras $\mathcal{O}_{q}\left(\mathrm{SL}_{n}\right), \mathcal{O}_{q}(P), \mathcal{O}_{q}\left(\mathrm{SL}_{n} / P\right)$ and their localizations are left and right (graded) $\mathcal{O}\left(\mathbb{T}^{n-1}\right)$-comodule algebras.

We first observe that the total space (global sections) of $\Sigma \circ \Gamma \circ \mathcal{F}$ is the multiparametric quantum group

$$
(\Sigma \circ \Gamma \circ \mathcal{F})\left(\mathrm{SL}_{n}(\mathbb{C})\right)=\mathcal{O}_{q, \gamma}\left(\mathrm{SL}_{n}\right)
$$

with $\mathcal{O}_{q, \gamma}(P)$ that is a quantum subgroup. Indeed we can pullback the twist (31) on $K=\mathcal{O}\left(\mathbb{T}^{n-1}\right)$ to the twist (33) on $\mathcal{O}_{q}\left(\mathrm{SL}_{n}\right)$. Then $(\Gamma \circ \Sigma)\left(\mathcal{O}_{q}\left(\mathrm{SL}_{n}\right)\right)$ is the twist of $\mathcal{O}_{q}\left(\mathrm{SL}_{n}\right)$ as a left $\mathcal{O}_{q}\left(\mathrm{SL}_{n}\right)$-comodule algebra and with the same twist (33) as a right $\mathcal{O}_{q}\left(\mathrm{SL}_{n}\right)$-comodule algebra, hence it is the twisting of $\mathcal{O}_{q}\left(\mathrm{SL}_{n}\right)$ as a Hopf algebra, giving the Hopf algebra $\mathcal{O}_{q, \gamma}\left(\mathrm{SL}_{n}\right)$. Similarly we have

$$
(\Sigma \circ \Gamma)\left(\mathcal{O}_{q}(P)\right)=\mathcal{O}_{q, \gamma}(P) .
$$

In order to show that $\mathcal{O}_{q, \gamma}(P)$ is a quantum subgroup of $\mathcal{O}_{q, \gamma}\left(\mathrm{SL}_{n}\right)$ recall that the deformation (34) is induced from a left and right action of the Hopf algebra $\mathcal{O}\left(\mathbb{T}^{n-1}\right)$ and notice that the ideal $I_{q}(P)=\left(a_{\alpha 1}\right) \subset \mathcal{O}_{q}\left(\mathrm{SL}_{n}\right)$ is a left and right $\mathcal{O}\left(\mathbb{T}^{n-1}\right)$ subcomodule algebra. Its twist deformation $I_{q, \gamma}(P):=(\Sigma \circ \Gamma)\left(I_{q}(P)\right)$ is an ideal in $\mathcal{O}_{q, \gamma}\left(\mathrm{SL}_{n}\right)$. It is furthermore a Hopf ideal since so was $I_{q}(P)$ in $\mathcal{O}_{q}\left(\mathrm{SL}_{n}\right)$, and because 
twisting does not affect the costructures and twisting via the exponential 2-cocycle (31) does not affect the antipode as a linear map. We can then consider the quotient Hopf algebra $\mathcal{O}_{q, \gamma}\left(\mathrm{SL}_{n}\right) / I_{q, \gamma}(P)$, this is easily seen to be the multiparametric quantum group in (35).

We next twist $\tilde{\mathcal{O}}_{q}\left(\mathbf{P}^{n-1}\right)=\tilde{\mathcal{O}}_{q}\left(\mathrm{SL}_{n} / P\right)$ seen as left $K=\mathcal{O}\left(\mathbb{T}^{n-1}\right)$-comodule algebra (and a trivial right $\mathcal{O}\left(\mathbb{T}^{n-1}\right)$-comodule algebra). The twist is grade preserving and therefore $\tilde{\mathcal{O}}_{q, \gamma}\left(\mathbf{P}^{n-1}\right):=(\Sigma \circ \Gamma)\left(\tilde{\mathcal{O}}_{q}\left(\mathbf{P}^{n-1}\right)\right)$ is a graded algebra. It is generated by the quantum section $d=a_{11} \in \tilde{\mathcal{O}}_{q, \gamma}\left(\mathrm{SL}_{n}\right)$ and the corresponding $d_{i}=a_{i 1}$ obtained from the coproduct (that equals that of $\mathcal{O}_{q}\left(\mathrm{SL}_{n}\right)$ ). Indeed monomials in $d_{i}$, respectively contructed with the product of $\tilde{\mathcal{O}}_{q}\left(\mathbf{P}^{n-1}\right)$ and of $\tilde{\mathcal{O}}_{q, \gamma}\left(\mathbf{P}^{n-1}\right)$, differ by a phase and hence span the same $\mathbb{C}$-module $\tilde{\mathcal{O}}_{q, \gamma}\left(\mathbf{P}^{n-1}\right)$. Explicitly $\tilde{\mathcal{O}}_{q, \gamma}\left(\mathbf{P}^{n-1}\right)$ is the subalgebra generated by the elements $x_{i}:=d_{i}=a_{i 1} \in \mathcal{O}_{q, \gamma}\left(\mathrm{SL}_{n}\right)$, i.e. it is the multiparametric quantum homogeneous projective space

$$
\tilde{\mathcal{O}}_{q, \gamma}\left(\mathbf{P}^{n-1}\right)=\mathbb{C}_{q}\left\langle x_{1}, \ldots x_{n}\right\rangle /\left(x_{i} x_{j}-q^{-1} \gamma_{i j}^{2} x_{j} x_{i}, i<j\right) .
$$

We now observe that $\mathcal{O}_{q}\left(\mathrm{SL}_{n}\right) S_{i}^{-1}$ is canonically an $\mathcal{O}\left(\mathbb{T}^{n-1}\right)$-bicomodule algebra. We twist it to $(\Sigma \circ \Gamma)\left(\mathcal{O}_{q}\left(\mathrm{SL}_{n}\right) S_{i}^{-1}\right)$ and denote by $\gamma \bullet \gamma$ the corresponding product (notice that $\gamma \bullet \gamma$ restricted to the sub $\mathcal{O}\left(\mathbb{T}^{n-1}\right)$-bicomodule $\mathcal{O}_{q}\left(\mathrm{SL}_{n}\right)$ is the Hopf algebra twist of the product of $\left.\mathcal{O}_{q}\left(\mathrm{SL}_{n}\right)\right)$. Due to $\gamma\left(t_{i}^{-1} \otimes t_{i}\right)=1=\gamma\left(t_{i} \otimes t_{i}^{-1}\right)$ (cf. (31) and (32)), we have $d_{i}^{-1}{ }^{\bullet} \bullet_{\gamma} d_{i}=d_{i}^{-1} d_{i}$ and $d_{i} \bullet^{\bullet} d_{i}^{-1}=d_{i} d_{i}^{-1}$. This shows that the inverse $d_{i}^{-1}$ of $d_{i}$ in $\mathcal{O}_{q}\left(\mathrm{SL}_{n}\right)$ is also the inverse in $\mathcal{O}_{q, \gamma}\left(\mathrm{SL}_{n}\right)$.

Then, the identity $\left(a_{\gamma} \bullet d_{i}^{-1}\right)_{\gamma} \bullet \gamma d_{i}=a_{\gamma} \bullet_{\gamma}\left(d_{i}^{-1}{ }_{\gamma} \bullet d_{i}\right)=a_{\gamma} \bullet \gamma\left(d_{i}^{-1} d_{i}\right)=a$, where $a \in \mathcal{O}_{q}\left(\mathrm{SL}_{n}\right)$, and more in general $a \in\left(\mathcal{O}_{q}\left(\mathrm{SL}_{n}\right) S_{i_{1}}^{-1} \ldots \widehat{S_{i}^{-1}} \ldots S_{i_{s}}^{-1}\right)_{\gamma}$, shows that the twist of the localizations of $\mathcal{O}_{q}\left(\mathrm{SL}_{n}\right)$ are just the localizations of the twisted quantum group $\mathcal{O}_{q, \gamma}\left(\mathrm{SL}_{n}\right)$, i.e.,

$$
(\Sigma \circ \Gamma \circ \mathcal{F})\left(U_{I}\right):=(\Sigma \circ \Gamma)\left(\mathcal{O}_{q}\left(\mathrm{SL}_{n}\right) S_{i_{1}}^{-1} \ldots S_{i_{s}}^{-1}\right)=\mathcal{O}_{q, \gamma}\left(\mathrm{SL}_{n}\right) S_{i_{1}}^{-1} \ldots S_{i_{s}}^{-1}
$$

$I=\left\{i_{1}, \ldots i_{s}\right\}, i_{1}, \ldots i_{s}, s \in\{1, \ldots n\}$. This shows that the Ore conditions are satisfied for the localizations of $\mathcal{O}_{q, \gamma}\left(\mathrm{SL}_{n}\right)$ and that the corresponding sheaf constructed as in Theorem 4.8 is $\Sigma \circ \Gamma \circ \mathcal{F}$. We summarize this result in the following theorem.

Theorem 6.4. The assignment:

$$
U_{I} \mapsto(\Sigma \circ \Gamma \circ \mathcal{F})\left(U_{I}\right)=\mathcal{O}_{q, \gamma}\left(\mathrm{SL}_{n}\right) S_{i_{1}}^{-1} \ldots S_{i_{s}}^{-1}, \quad I=\left\{i_{1}, \ldots, i_{s}\right\}
$$

$i_{1}, \ldots i_{s}, s \in\{1, \ldots n\}$, defines a locally cleft quantum principal bundle on the quantum ringed space $\left(\mathrm{SL}_{n}(\mathbb{C}) / P,(\Sigma \circ \Gamma \circ \mathcal{F})^{\mathrm{co}_{q, \gamma}(P)}\right)$, with structure sheaf $(\Sigma \circ \Gamma \circ \mathcal{F})^{\mathrm{co} \mathcal{O}_{q, \gamma}(P)}$ given by projective localizations of the multiparametric quantum homogeneous projective space $\tilde{\mathcal{O}}_{q, \gamma}\left(\mathbf{P}^{n-1}\right)=\tilde{\mathcal{O}}_{q, \gamma}\left(\mathrm{SL}_{n} / P\right)$.

Remark 6.5. An immediate corollary is that the $\mathcal{O}_{q, \gamma}(P)$-principal bundle $\Gamma \circ \Sigma \circ \mathcal{F}$ is locally trivial with cleaving maps $(\Sigma \circ \Gamma)\left(j_{i}\right): \mathcal{O}_{q, \gamma}(P) \rightarrow(\Sigma \circ \Gamma \circ \mathcal{F})\left(U_{i}\right)$ that are algebra maps (recall Remark 2.5). Indeed $j_{i}: \mathcal{O}_{q}(P) \rightarrow \mathcal{F}\left(U_{i}\right)$ in Proposition 5.7 are $\left(\mathcal{O}\left(\mathbb{T}^{n-1}\right), \mathcal{O}_{q}(P)\right)$-bicomodule algebra maps, and the result follows applying the functor $\Sigma \circ \Gamma$ and recalling (35). 
Remark 6.6. Applying Remark 6.2 (with the functor $\Gamma^{-1}$ instead of $\Gamma$ ) to the locally trivial bundle $\Gamma \circ \Sigma \circ \mathcal{F}=\Sigma \circ \Gamma \circ \mathcal{F}$ we conclude that the extensions $(\Sigma \circ \mathcal{F})\left(U_{i}\right)^{\operatorname{co} \mathcal{O}_{q}(P)} \subset$ $(\Sigma \circ \mathcal{F})\left(U_{i}\right)$ are cleft and nontrivial. So that the $\mathcal{O}_{q}(P)$-principal bundle $\Sigma \circ \mathcal{F}$ locally is cleft and nontrivial.

Acknowledgements. The authors wish to thank Prof. T. Brzeziński, Prof. F. Gavarini, Prof. T. Lenagan, Prof. Z. Škoda and Dr. C. Pagani for helpful comments. They also would like to thank the referees for their valuable observations. P.A. wishes to thank the Dipartimento di Matematica, Università di Bologna for the hospitality during the collaboration. R.F. and E.L. wish to thank the Dipartimento di Scienze e Innovazione Tecnologica, Università del Piemonte Orientale, Alessandria, for the hospitality during the collaboration. The work of P.A. is partially supported by INFN, CSN4, Iniziativa Specifica GSS, and by Università del Piemonte Orientale. P.A. is also affiliated to INdAM, GNFM (Istituto Nazionale di Alta Matematica, Gruppo Nazionale di Fisica Matematica).

Funding Open access funding provided by Università degli Studi del Piemonte Orientale Amedeo Avogrado within the CRUI-CARE Agreement.

Open Access This article is licensed under a Creative Commons Attribution 4.0 International License, which permits use, sharing, adaptation, distribution and reproduction in any medium or format, as long as you give appropriate credit to the original author(s) and the source, provide a link to the Creative Commons licence, and indicate if changes were made. The images or other third party material in this article are included in the article's Creative Commons licence, unless indicated otherwise in a credit line to the material. If material is not included in the article's Creative Commons licence and your intended use is not permitted by statutory regulation or exceeds the permitted use, you will need to obtain permission directly from the copyright holder. To view a copy of this licence, visit http://creativecommons.org/licenses/by/4.0/.

Publisher's Note Springer Nature remains neutral with regard to jurisdictional claims in published maps and institutional affiliations.

\section{References}

1. Artin, M., Zhang, J.J.: Noncommutative projective schemes. Adv. Math. 109, 228-287 (1994)

2. Aschieri, P., Bieliavsky, P., Pagani, C., Schenkel, A.: Noncommutative principal bundles through twist deformation. Commun. Math. Phys. 352, 287-344 (2017)

3. Borel, A.: Linear Algebraic Groups. Springer, New York (1991)

4. Brzeziński, T., Hajac, P.M.: The Chern-Galois character. C. R. Acad. Sci. Paris 338, 113-116 (2004)

5. Brzeziński, T., Janelidze, G., Maszczyk, T.: Galois structures. In: Hajac, P. M.(ed.) Lecture Notes on Noncommutative Geometry and Quantumcipa Groups. http://www.mimuw.edu.pl/pwit/toknotes/toknotes

6. Brzeziński, T., Majid, S.: Quantum group gauge theory on quantum spaces. Commun. Math. Phys. 157, 591-638 (1993)

7. Brzeziński, T., Fairfax, S.A.: Bundles over quantum real weighted projective spaces. Axioms 1, 201-225 (2015)

8. Cirio, L.S., Pagani, C.: A 4-sphere with non-central radius and its instanton sheaf. Lett. Math. Phys. 105, 169-197 (2015)

9. Carmeli, C., Caston, L., Fioresi, R.: Mathematical Foundation of Supersymmetry, with an appendix with I. Dimitrov. EMS Series of Lectures in Mathematics. European Mathematical Society, Zurich (2011)

10. Ciccoli, N., Fioresi, R., Gavarini, F.: Quantization of projective homogeneous spaces and duality principle. J. Noncommut. Geom. 2, 449-496 (2008)

11. D'Andrea, F., Dabrowski, L.: Dirac operators on quantum projective spaces. Commun. Math. Phys. 295, 731-790 (2010)

12. D’Andrea, F., Landi, G.: Quantum weighted projective and lens spaces. Commun. Math. Phys. 340, 325-353 (2015)

13. Doi, Y., Takeuchi, M.: Cleft comodule algebras for a bialgebra. Commun. Algebra 14, 801-818 (1986)

14. Eisenbud, D., Harris, J.: The Geometry of Schemes. Springer, New York (2000)

15. Fioresi, R.: Quantum deformation of the Grassmannian manifold. J. Algebra 214, $418-447$ (1999)

16. Fioresi, R.: Commutation relations among generic quantum minors in $O_{q}\left(M_{n}(k)\right)$. J. Algebra 280, 655682 (2004)

17. Fioresi, R., Gavarini, F.: Quantum duality principle for quantum Grassmannians. In: Marcolli, M., Parashar, D. (eds.) Quantum Groups and Noncommutative Spaces, pp. 80-95. Springer, Berlin (2011) 
18. Fioresi, R., Hacon, C.: Quantum coinvariant theory for the quantum special linear group and quantum Schubert varieties. J. Algebra 242, 433-446 (2001)

19. Kontsevich, M., Rosenberg, A.L.: Noncommutative Smooth Spaces, The Gelfand Mathematical Seminars, 1996-1999, pp. 85-108. Birkhäuser Boston, Boston, MA (2000)

20. Goodearl, K., Lenagan, T.: Quantized coinvariants at trascendental q. In: Hopf Algebras in Non Commutative Geometry and Physics, Lecture Notes in Pure and Applied Mathematics, vol. 239, pp. 155-165. Dekker (2005)

21. Griffiths, P., Harris, J.: Principles of Algebraic Geometry. Wiley, New York (1994)

22. Grothendieck, A.: A General Theory of Fibre Spaces with Structure Sheaf, Report No. 4, (Lawrence, Kansas) (1965)

23. Hajac, P.M., Krähmer, U., Matthes, R., Zieliński, B.: Piecewise principal comodule algebras. J. Noncommut. Geom. 5, 591-614 (2011)

24. Hajac, P.M., Nest, R., Pask, D., Sims, A., Zieliński, B.: The K-theory of twisted multipullback quantum odd spheres and complex projective spaces. J. Noncommut. Geom. 12, 823-863 (2018)

25. Hartshorne, R.: Algebraic Geometry. Graduate Text In Mathematics. Springer, New York (1977)

26. Heckenberger, I., Kolb, S.: The locally finite part of the dual coalgebra of quantised irreducible flag manifolds. Proc. Lond. Math. Soc. 89, 457-484 (2004)

27. Heckenberger, I., Kolb, S.: De Rham complex for quantized irreducible flag manifolds. J. Algebra 305, 704-741 (2006)

28. Khalkhali, M., Landi, G., van Suijlekom, W.: Holomorphic structures on the quantum projective line. Int. Math. Res. Not. 4, 851-884 (2011)

29. Landi, G., Zampini, A.: Calculi, Hodge operators and Laplacians on a quantum Hopf fibration. Rev. Math. Phys. 23, 575-613 (2011)

30. Manin, Y.I.: Topics in Noncommutative Geometry. M. B. Porter Lectures. Princeton University Press, Princeton, NJ (1991)

31. Montgomery, S.: Hopf Algebras and Their Actions on Rings, CBMS no. 82, AMS ed., Providence, RI (1993)

32. Montgomery, S., Schneider, H.-J.: Krull relations in Hopf Galois extensions: lifting and twisting. J. Algebra 288, 364-383 (2005)

33. Mrozinski, C., Ó Buachalla, R.: A Borel-Weil Theorem for the Quantum Grassmannians. arXiv:1611.07969 [math.QA]

34. Buachalla, R.Ó.: Quantum bundle description of quantum projective spaces. Commun. Math. Phys. 316, 345-373 (2012)

35. Reshetikhin, N.: Multiparameter quantum groups and twisted quasitriangular Hopf algebras. Lett. Math. Phys. 20, 331 (1990)

36. Rosenberg, A.L.: Noncommutative Algebraic Geometry and Representations of Quantized Algebras, Mathematics and its Applications, vol. 330. Kluwer Academic Publishers Group, Dordrecht (1995)

37. Parshall, B., Wang, J.: Quantum Linear Groups. Memoirs AMS 439 (1991)

38. Pflaum, M.: Quantum groups on fiber bundles. Commun. Math. Phys. 166, 279-315 (1994)

39. Škoda, Z.: Every quantum minor generates an Ore set. Int. Math. Res. Not. 2008, Article ID rnn063 (2008)

40. Škoda, Z.: Localizations for construction of quantum coset spaces. In: Pusz, W., Hajac, P.M. (eds.) Noncommutative Geometry and Quantum Groups, vol. 61, pp. 265-298. Banach Center Publications, Warszawa (2003)

41. Van Oystaeyen, F.M.J., Verschoren, A.H.M.J.: Non-commutative Algebraic Geometry. Lecture Notes in Mathematics, vol. 887. Springer, Berlin (1981)

Communicated by C. Schweigert 